FedUni ResearchOnline

https://researchonline.federation.edu.au

This is the published version of the following publication:

Ooi, E. T., Song, C., \& Natarajan, S. (2018). A scaled boundary finite element formulation with bubble functions for elasto-static analyses of functionally graded materials. Computational Mechanics, 60(6),

943-967.

The version displayed here may differ from the final published version.

The final publication is available at:

https://doi.org/10.1007/s00466-017-1443-y

Copyright (C) 2018, Springer. 


\title{
A Scaled Boundary Finite Element Formulation with Bubble Functions for Elasto-static Analyses of Functionally Graded Materials
}

\author{
E. T. Ooi* ${ }^{*}$ C. Song ${ }^{\dagger}$ and S. Natarajan ${ }^{\ddagger}$
}

Received: date / Accepted: date

\begin{abstract}
This manuscript presents an extension of the recently-developed high order complete scaled boundary shape functions to model elasto-static problems in functionally graded materials. Both isotropic and orthotropic functionally graded materials are modelled. The high order complete properties of the shape functions are realized through the introduction of bubble-like functions derived from the equilibrium condition of a polygon subjected to body loads. The bubble functions preserve the displacement compatibility between the elements in the mesh. The heterogeneity resulting from the material gradient introduces additional terms in the polygon stiffness matrix that are integrated analytically. Few numerical benchmarks were used to validate the developed formulation. The high order completeness property of the bubble functions result in superior accuracy and convergence rates for generic elasto-static and fracture problems involving functionally graded materials. Keywords: functionally graded materials; scaled boundary finite element method; shape functions; Bubble functions; polygon elements; heterogeneous
\end{abstract}

\section{Introduction}

Functionally graded materials (FGM) belong to the family of composite materials in which its material characteristics have been tailored to adapt to suit the environment and economic challenges in a specific application. Their microstructure comprise of two or more material phases, which vary continuously over a predefined volume. The continuously varying material properties eliminates sharp discontinuities at the material interfaces, which often resulted in stress concentrations in layered composites that leads to failure(Williamson et al, 1993). Owing to this characteristic, FGMs are particularly efficient at optimizing the stress distribution in a structure making them suitable for lightweight applications that require simultaneous resistance to high temperature and wear such as that found in the automotive, aerospace, biomedical and micro-electrics industries (Koizumi, 1997).

The behaviour of FGMs under loads is more complicated than that in homogeneous materials. The material gradients in FGMs affects the resulting stress distribution. Analytical solutions, although available e.g. (Kim and Paulino, 2002; Saddd, 2005), are limited to simple geometries, loading conditions and material gradients. Computational analysis have the potential to be an effective alternative to analyze and design components manufactured by FGMs. In this respect, computational approaches that are based on the finite element method (FEM), boundary element method (BEM), meshless methods and scaled boundary finite element method (SBFEM) has been investigated to some extent.

The most straightforward approach to model the material gradient in the FEM is to assign the appropriate material properties to each individual element (Enab, 2014) or a subdividion of elements in the mesh (Bao and Wang, 1995). This approach is not particularly efficient because the mesh density

\footnotetext{
E. T. Ooi*

*School of Engineering \& Information Technology, Federation University of Australia, Ballarat, VIC 3350, Australia.

E-mail: ooi.ean.tat@gmail.com.

C. Song ${ }^{\dagger}$

†School of Civil \& Environmental Engineering, The University of New South Wales, Sydney, NSW 2031, Australia.

E-mail: c.song@unsw.edu.au.

S. Natarajan ${ }^{\ddagger}$

${ }_{\ddagger}^{\ddagger}$ Department of Mechanical Engineering, Indian Institute of Technology, Madras, Chennai - 600036 .

E-mail: snatarajan@cardiffalumni.org.uk; snatarajan@iitm.ac.in.
} 
has to be sufficiently fine to keep the discontinuous step-type variation in the material properties to a minimum. An alternative approach that does not require any reformulation of the FEM was proposed by Rosseau and Tippur (2000). In this approach, the spatial variation of the material properties are defined as a function of temperature with zero thermal coefficient of expansion. An initial temperature gradient that matches the material gradient of the FGM is then specified to the mesh so that the resulting material gradients can be modelled. This approach can be adapted to commercial finite element softwares such as ANSYS and ADINA. In ABAQUS, the material gradient can be modelled using user defined subroutines such as UMAT e.g. (Berlo, 2009) or USDFLD e.g. (Martinez-Paneda and Gallego, 2015).

The approaches that require modifications the FEM formulation include the work of Santare and Lambros (2000) and the isoparametric graded element of Kim and Paulino (2002). Both approaches are equivalent and differs only in the manner in which the material gradient is incorporated into the formulation. In the former, the material properties at the integration points are directly interpolated from the material gradient. The latter uses the standard isoparametric finite element shape functions to interpolate the material gradient from their nodal values during the integration of the element stiffness matrix. The philosophy underlying the isoparametric graded formulation can also be adapted to polygonal meshes by adopting the polygonal interpolants e.g. Wachspress (1975); Meyer et al (2002); Sukumar and Tabarraei (2004). This was adopted by Vatanabe et al (2013) for topology optimization of functionally graded piezoelectric composites. Hernik (2010) proposed a further modification to the isoparametric graded formulation by interpolating the material gradient using special functions tailored to suit the material variation.

The BEM posseses many characteristics that makes it an appealing choice to be used for engineering analyses. It requires only the discretization of the boundary of the problem domain and therefore reduces the required spatial dimension of a generic problem by one compared with the FEM. This results in significant reduction in the required number of degrees-of-freedom leading to huge savings in computational cost. Several computational problems in the BEM that impede its application is the availability of a fundamental solution and the need to evaluate hypersingular integrals. Several approaches have been proposed to circumvent these difficulties. These include formulating the BEM in conjunction with a meshless scheme (Gao, 2002; Zhang et al, 2011; Yang et al, 2015), combining the BEM with analog equations (Riveiro and Gallego, 2013) and improved integration methodologies (Sladek and Sladek, 1998; Liu et al, 2015).

The application of the meshless method in computational mechanics became popular during the mid-1990's. Many variants of the meshless method have been introduced including for example, the Element-Free Galerkin method (Belytschko et al, 1994), the Reproducing Kernel Particle method (Liu et al, 1995) and the meshless local Petrov Galerkin method (Sladek et al, 2013). A detailed review of the meshless method and its implementation aspects is documented by Nguyen et al (2008). Unlike the FEM or the BEM, the meshless methods enable the solution of a generic boundary value problem without the definition of a physical mesh. This significantly simplifies the preprocessing phase in numerical simulations. The meshless methods have also been formulated for application in FGMs. Applications reported in the literature include anisotropic FGMS (Sladek et al, 2005), piezoelectric FGMS (Sladek et al, 2007), fracture (Sladek et al, 2008a,b), thermo-mechanical analysis of FGMs (Wang and Qin, 2008), magneto-elastic FGMs (Sladek et al, 2010) and viscoelastic FGMs (Chen et al, 2015).

The SBFEM is a semi-analytical method that was developed by Song and Wolf (1997) and is well known for its robustness in problems involving fracture e.g. (Goswami and Becker, 2012; Ooi et al, 2012; Natarajan et al, 2014) and unbounded media (Song and Tao, 2010; Birk and Behnke, 2012). A SBFEM formulation for FGMs was developed by Chiong et al (2014). The key step in this development is the reformulation of the SBFEM in the form of shape functions valid for arbitrary sided star convex polygons. The scaled boundary shape functions enable the modelling of general material heterogeneity and paved the way for modelling material nonlinearity (Ooi et al, 2014; Behnke et al, 2014). The scaled boundary shape functions possess linear completeness properties, which guarantees convergence in the limit when the size of the element approaches zero (Chiong et al, 2014).

Ooi et al (2016) recently showed that the scaled boundary shape functions do not possess high order completeness, which resulted in sub-optimal convergence in modal analyses and problems of the Poisson type in homogeneous materials. A set of high order complete shape functions was then developed by introducing bubble-like functions to the original SBFEM interpolants. The bubble functions are derived from the scaled boundary formulation by incorporating body force modes using a generalized coordinates (Song, 2006). Unlike the conventional bubble functions in the context of the FEM (Taylor et al, 1976), those derived by Ooi et al (2016) naturally preserve the displacement compatibility between adjacent polygons without any remedial techniques e.g. (Ibrahimbegovic and Wilson, 1991). Higher 
order interpolations can be conveniently formulated by simultaneously increasing the order of the onedimensional shape functions on the polygon boundary and the number of the body force modes. This manuscript extends the high order complte SBFEM formulation with bubble functions developed by Ooi et al (2016) for elasto-static analyses of FGMs.

This manuscript is organized as follows: In Section 2, the fundamentals of the SBFEM in in terms of generalized coordinates (Song, 2006) are reviewed; leading to the derivation of the bubble-like shape functions. Section 3 outlines the formulation of the stiffness matrix for a generic polygon applicable to isotropic and orthotropic FGMs. A series of numerical benchmarks used to validate the developed formulation is presented in Section 4. The accuracy of the developed formulation is compared with available analytical solutions reported in the literature. Section 5 summarizes the major conclusions that can be drawn from this manuscript.

\section{Scaled boundary finite element formulation with bubble functions}

\subsection{Geometry transformation}

Any star-convex polygon can be modelled using the SBFEM. A generic polygon modelled by the SBFEM is shown in Fig. 1a. The star convexity criterion necessitates the definition of a point inside a polygon known as the scaling centre from which every point on the boundary of the polygon is visible from it. A radial coordinate $\xi$ is defined in the polygon where $\xi=0$ at the scaling centre and $\xi=1$ at the polygon boundary. The edges of a polygon are discretized using one-dimensional finite elements. A local coordinate system $\eta$ similar to that in the FEM; with $-1 \leq \eta \leq 1$ is defined on each line element. Along the line elements, standard finite element shape functions can be used to approximate the field variable. The SBFEM can also be used to model discontinuities such as a crack or a notch. When modelling such types of geometries, the polygon does not form a closed loop and the scaling centre is selected to be the crack/notch tip. A generic polygon modelling a crack is shown in Fig. 1b.

With reference to the scaling centre, the Cartesian coordinates of a point in the polygon is

$$
\left\{\begin{array}{l}
x(\xi, \eta) \\
y(\xi, \eta)
\end{array}\right\}=\xi\left\{\begin{array}{l}
x_{\eta} \\
y_{\eta}
\end{array}\right\}
$$

where

$$
\left\{\begin{array}{l}
x_{\eta} \\
y_{\eta}
\end{array}\right\}=\mathbf{N}(\eta) \mathbf{x}_{0}
$$

In Eq. $(2), \mathbf{N}(\eta)$ is the shape function matrix. For a polygon with $m$ nodes, $\mathbf{N}(\eta)$ is

$$
\mathbf{N}(\eta)=\left[\begin{array}{ccccccc}
N_{1}(\eta) & 0 & N_{2}(\eta) & 0 & \ldots & N_{m}(\eta) & 0 \\
0 & N_{1}(\eta) & 0 & N_{2}(\eta) & \ldots & 0 & N_{m}(\eta)
\end{array}\right]
$$

and $\mathbf{x}_{0}$ is the vector of nodal coordinates

$$
\mathbf{x}_{0}=\left[\begin{array}{lllllll}
x_{1} & y_{1} & x_{2} & y_{2} & \ldots & x_{m} & y_{m}
\end{array}\right]^{T}
$$

The coordinate transformation between the scaled boundary coordinates $(\xi, \eta)$ and the Cartesian coordinates $(x, y)$ is performed similar to the FEM. The Jacobian matrix required for coordinate transformation is (Song, 2006)

$$
\mathbf{J}(\eta)=\left[\begin{array}{cc}
x_{\eta} & y_{\eta} \\
x_{\eta}, \eta & y_{\eta}, \eta
\end{array}\right]
$$

Using Eq. (5), the derivatives with respect to $x$ and $y$ is transformed to those with respect to $\xi$ and $\eta$ as

$$
\left\{\begin{array}{c}
\frac{\partial}{\partial x} \\
\frac{\partial}{\partial y}
\end{array}\right\}=\frac{1}{J}\left\{\begin{array}{c}
y_{\eta}, \eta \\
-x_{\eta}, \eta
\end{array}\right\} \frac{\partial}{\partial \xi}+\frac{1}{J} \xi^{-1}\left\{\begin{array}{c}
-y_{\eta} \\
x_{\eta}
\end{array}\right\} \frac{\partial}{\partial \eta}
$$


where $J$ is the determinant of the Jacobian matrix in Eq. (5). For a polygon, an infinitesimal area $d \Omega$ and an infinitesimal line on the polygon boundary $d \Gamma$ are expressed as (Song, 2006)

$$
\begin{aligned}
d \Omega & =J \xi d \xi d \eta \\
d \Gamma^{(\xi)} & =\Delta_{\Gamma} \xi d \eta
\end{aligned}
$$

where

$$
\Delta_{\Gamma}=\sqrt{x_{\eta}^{2}(\eta)+y_{\eta}^{2}(\eta)}
$$

2.2 High-order complete scaled boundary shape functions

\subsubsection{Static equilibrium condition within a polygon}

A set of higher order complete shape functions that is valid for an arbitrary sided star-convex polygon can be derived from the equilibrium conditions of the polygon (Ooi et al, 2016)

$$
\mathbf{L}^{T} \boldsymbol{\sigma}+\mathbf{p}=0
$$

where $\mathbf{p}$ is the body force intensity and the linear operator matrix $\mathbf{L}$ is defiend as (Song and Wolf, 1997)

$$
\mathbf{L}=\mathbf{b}_{1}(\eta) \frac{\partial}{\partial \xi}+\xi^{-1} \mathbf{b}_{2}(\eta) \frac{\partial}{\partial \eta}
$$

where

$$
\begin{aligned}
& \mathbf{b}_{1}(\eta)=\frac{1}{J}\left[\begin{array}{cc}
y_{\eta, \eta} & 0 \\
0 & -x_{\eta, \eta} \\
-x_{\eta, \eta} & y_{\eta}, \eta
\end{array}\right] \\
& \mathbf{b}_{2}(\eta)=\frac{1}{J}\left[\begin{array}{cc}
-y_{\eta} & 0 \\
0 & x_{\eta} \\
x_{\eta} & -y_{\eta}
\end{array}\right]
\end{aligned}
$$

The stress vector $\boldsymbol{\sigma}=\left[\begin{array}{lll}\sigma_{x x} & \sigma_{y y} & \tau_{x y}\end{array}\right]^{T}$ is related to the strain vector $\varepsilon=\left[\begin{array}{lll}\varepsilon_{x x} & \varepsilon_{y y} & \gamma_{x y}\end{array}\right]^{T}$ by the Hooke's law

$$
\sigma=\mathrm{D} \varepsilon
$$

where $\mathbf{D}$ is the material constitutive matrix. The strain vector $\boldsymbol{\epsilon}$ is related to the displacement vector by

$$
\varepsilon=\mathbf{L u}
$$

where $\mathbf{u}=\left[\begin{array}{ll}u & v\end{array}\right]^{T}$ is the displacement vector. For a point in a triangular sector bounded by the scaling centre and a line element on the polygon boundary, the displacement vector is interpolated as

$$
\mathbf{u}(\xi, \eta)=\mathbf{N}(\eta) \mathbf{u}(\xi)
$$

$\mathbf{u}(\xi)$ are analytical displacement functions that are obtained from the equilibrium condition of a polygon. Using Eq. (6), Eq. (16) and Eq. (15), the strain field can be expressed as

$$
\varepsilon(\xi, \eta)=\mathbf{B}_{1}(\eta) \frac{\partial \mathbf{u}(\xi)}{\partial \xi}+\xi^{-1} \mathbf{B}_{2}(\eta) \mathbf{u}(\xi)
$$

where

$$
\begin{aligned}
& \mathbf{B}_{1}(\eta)=\mathbf{b}_{1}(\eta) \mathbf{N}(\eta) \\
& \mathbf{B}_{2}(\eta)=\mathbf{b}_{2}(\eta) \mathbf{N}_{\eta}(\eta)
\end{aligned}
$$

Accordingly, the equilibrium equation can be expressed as

$$
\mathbf{b}_{1}^{T}(\eta) \frac{\partial \boldsymbol{\sigma}(\xi, \eta)}{\partial \xi}+\xi^{-1} \mathbf{b}_{2}^{T}(\eta) \frac{\partial \boldsymbol{\sigma}(\xi, \eta)}{\partial \eta}+\mathbf{p}(\xi, \eta)=0
$$


where it is assumed that the body force intensity can be expressed in terms of scaled boundary coordinates $(\xi, \eta)$.

The Galerkin weighted-residual technique can then be applied to solve Eq. (20). A weight function $\mathbf{w}(\xi, \eta)$ of the form

$$
\mathbf{w}(\xi, \eta)=\mathbf{N}(\eta) \mathbf{w}(\xi)
$$

is introduced. Multiplying Eq. (20) with $\mathbf{w}(\xi, \eta)$ and integrating over a triangular sector bounded by the scaling centre and a line element on the polygon boundary results in

$$
\begin{array}{r}
\int_{\Omega} \mathbf{w}(\xi)^{T} \mathbf{N}^{T}(\eta) \mathbf{b}_{1}^{T}(\eta) \boldsymbol{\sigma}(\xi, \eta)_{, \xi} d \Omega+\int_{\Omega} \mathbf{w}(\xi)^{T} \mathbf{N}^{T}(\eta) \xi^{-1} \mathbf{b}_{2}^{T}(\eta) \boldsymbol{\sigma}(\xi, \eta)_{, \eta} d \Omega \ldots \\
+\int_{\Omega} \mathbf{w}(\xi)^{T} \mathbf{N}^{T}(\eta) \mathbf{p}(\xi, \eta) d \Omega=0
\end{array}
$$

The second term in Eq. (22) is considered. Using the identity (Song, 2006)

$$
\left(J \mathbf{b}_{2}(\eta)\right)_{\eta}=-J \mathbf{b}_{1}(\eta)
$$

results in

$$
e=\int_{0}^{1} \mathbf{w}^{T}(\xi)\left(\mathbf{T}(\xi)-\int_{-1}^{1}\left(-\mathbf{B}_{1}^{T}(\eta)+\mathbf{B}_{2}^{T}(\eta)\right) \mathbf{D}\left(\mathbf{B}_{1}(\eta) \mathbf{u}(\xi), \xi+\xi^{-1} \mathbf{B}_{2}(\eta) \mathbf{u}(\xi)\right) J d \eta\right) d \xi
$$

where $\mathbf{T}(\xi)$ is the vector of nodal forces acting on the side-face of a the triangular sector at constant $\eta$. For closed loop polygons and crack polygons with traction free surfaces such as that dealt with in this manuscript, this term vanishes. Substituting this result into Eq. (22) and using Eq. (6) results in

$$
\int_{0}^{1} \mathbf{w}^{T}(\xi)\left(\mathbf{E}_{0} \xi \mathbf{u}(\xi)_{, \xi \xi}+\left(\mathbf{E}_{0}+\mathbf{E}_{1}^{T}-\mathbf{E}_{1}\right) \mathbf{u}(\xi)_{, \xi}-\mathbf{E}_{2} \xi^{-1} \mathbf{u}(\xi)+\int_{-1}^{1} \mathbf{N}^{T}(\eta) \mathbf{p}(\xi, \eta) J d \eta\right) \xi d \xi=0
$$

where the coefficient matrices $\mathbf{E}_{0}, \mathbf{E}_{1}$ and $\mathbf{E}_{2}$ are

$$
\begin{aligned}
& \mathbf{E}_{0}=\int_{-1}^{1} \mathbf{B}_{1}^{T}(\eta) \mathbf{D B}_{1}(\eta) J d \eta \\
& \mathbf{E}_{1}=\int_{-1}^{1} \mathbf{B}_{2}^{T}(\eta) \mathbf{D B}_{1}(\eta) J d \eta \\
& \mathbf{E}_{2}=\int_{-1}^{1} \mathbf{B}_{2}^{T}(\eta) \mathbf{D B}_{2}(\eta) J d \eta
\end{aligned}
$$

It is important to note here that the coefficient matrices $\mathbf{E}_{i}$ can be computed for each line element on the polygon boundary and assembled element by element similar to to the standard finite element procedure for assembly of the stiffness matrix.

Invoking the arbitrariness of the weight function, the equilibrium condition for a polygon can be expressed as

$$
\mathbf{E}_{0} \xi^{2} \mathbf{u}(\xi), \xi \xi+\left(\mathbf{E}_{0}+\mathbf{E}_{1}^{T}-\mathbf{E}_{1}\right) \xi \mathbf{u}(\xi)_{, \xi}-\mathbf{E}_{2} \mathbf{u}(\xi)+\mathbf{F}^{(b)}=0
$$

where $\mathbf{F}^{(b)}$ is the vector of nodal forces due to the body loads and can in general, be in polynomial form

$$
\mathbf{F}^{(b)}=\sum_{h=0} \sum_{k=0}^{h} \xi^{j+2} \mathbf{P}_{h k} \mathbf{a}_{h k}
$$

For a typical term with $h$ and $k$, the matrix $\mathbf{P}_{h k}$ is

$$
\mathbf{P}_{h k}=\int_{-1}^{1} \mathbf{N}^{T}(\eta) f_{h k}(\eta) J d \eta
$$

Eq. (25) is known as the scaled boundary finite element equation in displacement in the literature. 


\subsubsection{Solution of the scaled boundary finite element equation}

The system of linear nonhomogeneous second-order ordinary differential equations in Eq. (25) is solved by transforming it into a first-order ordinary differential equation with twice the number of unknowns (Song, 2006). The variable

$$
\chi(\xi)=\left\{\begin{array}{l}
\mathbf{u}(\xi) \\
\mathbf{q}(\xi)
\end{array}\right\}
$$

is introduced. Here, $\mathbf{q}(\xi)$ is the vector of nodal force functions on a surface $\Gamma^{(\xi)}$ with constant $\xi$. It is defined as(Song, 2006)

$$
\mathbf{q}(\xi)=\mathbf{E}_{0} \xi \mathbf{u}(\xi), \xi+\mathbf{E}_{1}^{T} \mathbf{u}(\xi)
$$

Using the definition of $\chi(\xi)$ in Eq. (32) together with Eq. (33) and Eq. (25) leads to

$$
\xi\left\{\begin{array}{l}
\mathbf{u}(\xi) \\
\mathbf{q}(\xi)
\end{array}\right\}, \xi=-\mathbf{Z}\left\{\begin{array}{c}
\mathbf{u}(\xi) \\
\mathbf{q}(\xi)
\end{array}\right\}-\left\{\begin{array}{c}
\mathbf{0} \\
\mathbf{F}^{(b)}
\end{array}\right\}
$$

where the Hamiltonian matrix

$$
\mathbf{Z}=\left[\begin{array}{cc}
\mathbf{E}_{0}^{-1} \mathbf{E}_{1}^{T} & \mathbf{E}_{0}^{-1} \\
-\mathbf{E}_{2}+\mathbf{E}_{1} \mathbf{E}_{0}^{-1} \mathbf{E}_{1}^{T} & -\mathbf{E}_{1} \mathbf{E}_{0}^{-1}
\end{array}\right]
$$

is of size $2 N \times 2 N$ and $N$ is the number of degrees-of-freedom in a polygon. The Hamiltonian matrix also satisfies the identity (Song, 2006)

$$
\left(\mathbf{J}_{2 n} \mathbf{Z}\right)^{T}=\mathbf{J}_{2 n} \mathbf{Z}
$$

where

$$
\mathbf{J}_{2 n}=\left[\begin{array}{cc}
\mathbf{0} & -\mathbf{I} \\
\mathbf{I} & \mathbf{0}
\end{array}\right]
$$

and $\mathbf{I}$ is an identity matrix.

The Hamiltonian matrix $\mathbf{Z}$ can be decomposed into base vectors using the Schur decomposition. This results in

$$
\mathbf{Z V}=\mathbf{V} \boldsymbol{\Lambda}
$$

where the Schur matrix $\boldsymbol{\Lambda}$ and $\mathbf{V}$ is a transformation matrix. The Schur matrix $\boldsymbol{\Lambda}$ is partitioned as (Song, 2006)

$$
\boldsymbol{\Lambda}=\operatorname{diag}\left(\boldsymbol{\Lambda}_{n}, \boldsymbol{\Lambda}_{z}, \boldsymbol{\Lambda}_{p}\right)
$$

with

$$
\begin{aligned}
& \boldsymbol{\Lambda}_{n}=\operatorname{diag}\left(\boldsymbol{\Lambda}_{1}, \ldots \boldsymbol{\Lambda}_{N-1}\right) \\
& \boldsymbol{\Lambda}_{z}=\left[\begin{array}{cc}
\boldsymbol{\Lambda}_{N} & \mathbf{0} \\
\mathbf{0} & \boldsymbol{\Lambda}_{N+1}
\end{array}\right] \\
& \boldsymbol{\Lambda}_{p}=\operatorname{diag}\left(\boldsymbol{\Lambda}_{N+2}, \ldots \boldsymbol{\Lambda}_{2 N}\right)
\end{aligned}
$$

The diagonal entries of $\boldsymbol{\Lambda}$ are equal to the real parts of the eigenvalues of $\mathbf{Z}$. In particular $\boldsymbol{\Lambda}_{n}$ is the diagonal block with negative eigenvalues, $\boldsymbol{\Lambda}_{z}$ is the diagonal block with zero eigenvalues and $\boldsymbol{\Lambda}_{p}$ is the diagonal block with positive eigenvalues. The $2 N$ diagonal blocks of $\boldsymbol{\Lambda}$ form $N$ pairs of conjugate $\boldsymbol{\Lambda}_{i}$ and $\boldsymbol{\Lambda}_{\bar{\imath}}$ where the index of the block conjugate to the ith block is

$$
\bar{\imath}=2 N+1-i
$$

The transformation matrix $\mathbf{V}$ has the form

$$
\mathbf{V}=\left[\mathbf{V}_{1}, \ldots, \mathbf{V}_{N-1}, \mathbf{V}_{N}, \mathbf{V}_{N+1}, \ldots \mathbf{V}_{2 N}\right]
$$


It can also be partitioned as

$$
\mathbf{V}=\left[\begin{array}{l}
\mathbf{V}^{(u)} \\
\mathbf{V}^{(q)}
\end{array}\right]=\left[\begin{array}{cc}
\mathbf{V}_{n}^{(u)} & \mathbf{V}_{p}^{(u)} \\
\mathbf{V}_{n}^{(q)} & \mathbf{V}_{p}^{(q)}
\end{array}\right]
$$

Each block $\mathbf{V}_{i}$ in Eq. (44) is $\mathbf{J}_{2 n}$-orthogonal (Song, 2006) i.e.

$$
\mathbf{V}_{i}^{T} \mathbf{J}_{2 n} \mathbf{V}_{j}=-\left(\mathbf{V}_{j}^{T} \mathbf{J}_{2 n} \mathbf{V}_{i}\right)^{T}=\left\{\begin{array}{cc}
\mathbf{H}_{i} & \text { when } j=\bar{\imath} \\
0 & \text { when } j \neq \bar{\imath}
\end{array}\right.
$$

Pre-multiplying Eq. (38) with $-\mathbf{V}_{\frac{T}{\imath}}^{T} \mathbf{J}_{2 n}$ and using Eq. (46) results in

$$
-\mathbf{V}_{\bar{\imath}}^{T} \mathbf{J}_{2 n} \mathbf{Z V}_{j}=\left\{\begin{array}{c}
\mathbf{H}_{i}^{T} \text { when } j=i \\
0 \quad \text { when } j \neq i
\end{array}\right.
$$

The solution of Eq. (34) is expressed in terms of generalized coordinates $\mathbf{W}(\xi)$ as (Song, 2006)

$$
\left\{\begin{array}{l}
\mathbf{u}(\xi) \\
\mathbf{q}(\xi)
\end{array}\right\}=\mathbf{V W}(\xi)
$$

The solution of $\mathbf{u}(\xi)$ depends on the the eigenvalues of the diagonal block matrices $\boldsymbol{\Lambda}$ in the Schur matrix.

For each of the the diagonal blocks $\boldsymbol{\Lambda}_{i}$ in $\boldsymbol{\Lambda}$, substituting Eq. (48) into Eq. (34) pre-multiplied by $-\mathbf{V}_{\bar{\imath}}^{T} \mathbf{J}_{2 n}$ and using the relations in Eq. (46) and Eq. (47) leads to

$$
\xi \mathbf{W}_{i}(\xi)_{, \xi}=-\boldsymbol{\Lambda}_{i} \mathbf{W}_{i}(\xi)-\mathbf{F}_{i}^{(w)}
$$

For each block $i, \mathbf{F}^{(w)}$ can be explicitly expressed as

$$
\mathbf{F}_{i}^{(w)}(\xi)=-\mathbf{H}_{i}^{-T}\left(\mathbf{V}_{\bar{\imath}}^{(u)}\right)^{T} \sum_{h=0} \sum_{k=0}^{h} \xi^{h+2}\left(\mathbf{P}_{h k}\right)_{i}\left(\mathbf{a}_{h k}\right)_{i}
$$

Solving Eq. 49 results in

$$
\mathbf{W}_{i}(\xi)=\xi^{-\Lambda_{i}} \mathbf{c}_{i}-\mathbf{W}_{i}^{(b)}(\xi)
$$

where $\mathbf{c}_{i}$ are the integration constants and for each of the diagonal block $i$, they are determined as

$$
\mathbf{c}_{i}=\int_{1}^{0} \tau^{\boldsymbol{\Lambda}_{i}-\mathbf{I}} \mathbf{F}_{i}^{(w)}(\tau) d \tau
$$

The first and second terms on the right-hand-side of Eq. (51) are the homogeneous solution and the particular solution, respectively. The particular solution is

$$
\mathbf{W}_{i}^{(b)}(\xi)=-\xi^{-\boldsymbol{\Lambda}_{i}} \int_{1}^{\xi} \tau^{\boldsymbol{\Lambda}_{i}-\mathbf{I}} \mathbf{F}_{i}^{(w)}(\xi) d \tau
$$

This solution is valid for any lower limit of the integral.

The particular solution is evaluated by substituting Eq. (50) into Eq. (52) and integrating analytically resulting in

$$
\mathbf{W}_{i}^{(b)}(\xi)=\left(\boldsymbol{\Lambda}_{i}+(h+2) \mathbf{I}\right)^{-1} \mathbf{H}_{i}^{-T}\left(\mathbf{V}_{\bar{\imath}}^{(u)}\right)^{T} \sum_{h=0} \sum_{k=0}^{h} \xi^{h+2}\left(\mathbf{P}_{h k}\right)_{i}\left(\mathbf{a}_{h k}\right)_{i}
$$

Substituting Eq. (54) into Eq. (51) and then the resulting equation into Eq. (48), the analytical displacement functions in Eq. (48) can be expressed as

$$
\mathbf{u}(\xi)=\mathbf{V}_{n}^{(u)} \xi^{-\boldsymbol{\Lambda}_{n}} \mathbf{c}+\mathbf{V}_{b} \xi^{\boldsymbol{\Lambda}_{b}} \mathbf{a}
$$

where

$$
\begin{aligned}
\mathbf{V}_{b} & =\mathbf{V}^{(u)}\left[\overline{\mathbf{W}}_{00}^{(b)} \overline{\mathbf{W}}_{10}^{(b)} \overline{\mathbf{W}}_{11}^{(b)} \overline{\mathbf{W}}_{20}^{(b)} \overline{\mathbf{W}}_{21}^{(b)} \overline{\mathbf{W}}_{22}^{(b)} \ldots\right] \\
\mathbf{a} & =\left[\mathbf{a}_{00} \mathbf{a}_{10} \mathbf{a}_{11} \mathbf{a}_{20} \mathbf{a}_{21} \mathbf{a}_{22} \ldots\right]^{T} \\
\boldsymbol{\Lambda}_{b} & =\operatorname{diag}(2,3,3,4,4,4, \ldots)
\end{aligned}
$$


For each block $i, \overline{\mathbf{W}}_{h k}^{(b)}$ in Eq. (59) is expressed as

$$
\left(\overline{\mathbf{W}}_{h k}^{(b)}\right)_{i}=\left(\boldsymbol{\Lambda}_{i}+(h+2) \mathbf{I}\right)^{-1} \mathbf{H}_{i}^{-T}\left(\mathbf{V}_{\bar{\imath}}^{(u)}\right)^{T}\left(\mathbf{P}_{h k}\right)_{i}
$$

The integration constants $\mathbf{c}$ are determined from the nodal displacements $\mathbf{v}_{0}=\mathbf{u}(\xi=1)$ as

$$
\mathbf{c}=\left(\mathbf{V}_{n}^{(u)}\right)^{-1}\left(\mathbf{v}_{0}-\mathbf{V}_{b} \mathbf{a}\right)
$$

Substituting Eq. (60) into Eq. (55) and simplifying the equation results in

$$
\mathbf{u}(\xi)=\mathbf{V}_{n}^{(u)} \xi^{-\boldsymbol{\Lambda}_{n}}\left(\mathbf{V}_{n}^{(u)}\right)^{-1}\left(\mathbf{v}_{0}-\mathbf{V}_{b} \mathbf{a}\right)+\mathbf{V}_{b} \xi^{\boldsymbol{\Lambda}_{b}} \mathbf{a}
$$

or, in matrix form

$$
\mathbf{u}(\xi)=\left[\begin{array}{lll}
\mathbf{V}_{n}^{(u)} & \mathbf{V}_{b}
\end{array}\right]\left[\begin{array}{ll}
\xi^{-\Lambda_{n}} & \\
& \xi^{\Lambda_{b}}
\end{array}\right] \mathbf{T u}_{0}
$$

where

$$
\begin{aligned}
& \mathbf{T}=\left[\begin{array}{cc}
\left(\mathbf{V}_{n}^{(u)}\right)^{-1} & \left(\mathbf{V}_{n}^{(u)}\right)^{-1} \mathbf{V}_{b} \\
\mathbf{0} & \mathbf{I}
\end{array}\right] \\
& \mathbf{u}_{0}=\left[\begin{array}{ll}
\mathbf{v}_{0} & \mathbf{a}
\end{array}\right]^{T}
\end{aligned}
$$

By introducing the following notations

$$
\begin{aligned}
\boldsymbol{\Psi}_{u} & =\left[\begin{array}{ll}
\mathbf{V}_{n}^{(u)} & \mathbf{V}_{b}
\end{array}\right] \\
\mathbf{S} & =\left[\begin{array}{ll}
-\boldsymbol{\Lambda}_{n} & \\
& \boldsymbol{\Lambda}_{b}
\end{array}\right]
\end{aligned}
$$

the radial displacement functions $\mathbf{u}(\xi)$ can be further simplified as

$$
\mathbf{u}(\xi)=\boldsymbol{\Psi}_{u} \xi^{\mathbf{S}} \mathbf{T u}_{0}
$$

\subsubsection{Formulation of scaled boundary shape functions with bubble functions}

The complete displacement field of a point lying in a sector covered by a line element on the polygon boundary is obtained interpolating $\mathbf{u}(\xi)$ by $\mathbf{N}(\eta)$ as

$$
\mathbf{u}(\xi, \eta)=\mathbf{N}(\eta) \boldsymbol{\Psi}_{u} \xi^{\mathbf{S}} \mathbf{T} \mathbf{u}_{0}
$$

where the shape functions are defined in the matrix product $\mathbf{N}(\eta) \boldsymbol{\Psi}_{u} \xi^{\mathbf{S}} \mathbf{T}$. Using Eq. (65), Eq. (66) and Eq. (63), the shape functions can be expressed as

$$
\mathbf{N}(\eta) \boldsymbol{\Psi}_{u} \xi^{\mathbf{S}} \mathbf{T}=\left[\mathbf{N}(\eta) \mathbf{V}_{n}^{(u)} \xi^{-\boldsymbol{\Lambda}_{n}}\left(\mathbf{V}_{n}^{(u)}\right)^{-1} \mathbf{N}(\eta)\left(\mathbf{V}_{b} \xi^{\boldsymbol{\Lambda}_{b}}-\mathbf{V}_{n}^{(u)} \xi^{-\boldsymbol{\Lambda}_{n}}\left(\mathbf{V}_{n}^{(u)}\right)^{-1} \mathbf{V}_{b}\right)\right]
$$

The product of the matrices in the first term on the right-hand-side of Eq. (69) is the standard scaled boundary shape functions (Ooi et al, 2014). It is denoted as

$$
\boldsymbol{\Phi}(\xi, \eta)=\mathbf{N}(\eta) \mathbf{V}_{n}^{(u)} \xi^{-\Lambda_{n}}\left(\mathbf{V}_{n}^{(u)}\right)^{-1}
$$

The bubble functions $\boldsymbol{\Phi}^{(b)}(\xi, \eta)$ (Ooi et al, 2016) are defined in the second column in the of Eq. (69)

$$
\boldsymbol{\Phi}^{(b)}(\xi, \eta)=\mathbf{N}(\eta)\left(\mathbf{V}_{b} \xi^{\boldsymbol{\Lambda}_{b}}-\mathbf{V}_{n}^{(u)} \xi^{-\boldsymbol{\Lambda}_{n}}\left(\mathbf{V}_{n}^{(u)}\right)^{-1} \mathbf{V}_{b}\right)
$$

$\boldsymbol{\Phi}^{(b)}(\xi, \eta)$ is applicable to star convex polygons with an arbitrary number of sidesand it vanishes at the boundary $(\xi=1)$. For a typical term defined by the indices $h$ and $k$, the bubble function is

$$
\boldsymbol{\Phi}_{h k}^{(b)}(\xi, \eta)=\mathbf{N}(\eta)\left(\xi^{h+2} \mathbf{I}-\mathbf{V}_{n}^{(u)} \xi^{-\boldsymbol{\Lambda}_{n}}\left(\mathbf{V}_{n}^{(u)}\right)^{-1}\right) \mathbf{V}^{(u)} \overline{\mathbf{W}}_{h k}^{(b)}
$$

The index $h+2$ represents the order of the bubble function and the index $k$ corresponds to the specific mode that $\boldsymbol{\Phi}_{h k}^{(b)}(\xi, \eta)$ can represent. 


\section{Polygon formulation for functionally graded materials}

For problems in elasto-statics, the equilibrium condition of a domain $\Omega$ can be expressed using the principal of virtual work as

$$
\int \delta \varepsilon^{T} \boldsymbol{\sigma} d \Omega=\int_{\Gamma} \delta \mathbf{u}^{T} \mathbf{t} d \Gamma+\int_{\Omega} \delta \mathbf{u}^{T} \mathbf{b} d \Omega
$$

where $\boldsymbol{\sigma}$ is the stress field, $\boldsymbol{\varepsilon}$ is the strain field, $\mathbf{b}$ is the body force intensity and $\mathbf{t}$ is the surface traction.

\subsection{Stiffness matrix}

The stiffness matrix of a polygon $\mathbf{K}_{\text {pol }}$ is defined in the term on the left-hand-side of Eq. (73). The stress $\boldsymbol{\sigma}$ is related to the strain by Hooke's law in Eq. (14).

For functionally graded materials, the material constitutive matrix $\mathbf{D}$ is a function of the space $(x, y)$. For isotropic materials

$$
\mathbf{D}(x, y)=\left\{\begin{array}{c}
\frac{E(x, y)}{1-\nu(x, y)}\left[\begin{array}{ccc}
1 & \nu(x, y) & 0 \\
\nu(x, y) & 1 & 0 \\
0 & 0 & 1-\nu(x, y)
\end{array}\right] ; \quad \text { for plane stress } \\
\frac{E(x, y)}{(1+\nu(x, y))(1-2 \nu(x, y))}\left[\begin{array}{ccc}
1-\nu(x, y) & \nu(x, y) \\
\nu(x, y) & 1-\nu(x, y) & 0 \\
0 & 0 & 1-2 \nu(x, y)
\end{array}\right] ; \quad \text { for plane strain }
\end{array}\right.
$$

where $E(x, y)$ is the Young's modulus and $\nu(x, y)$ is the Poisson's ratio. For orthotropic materials

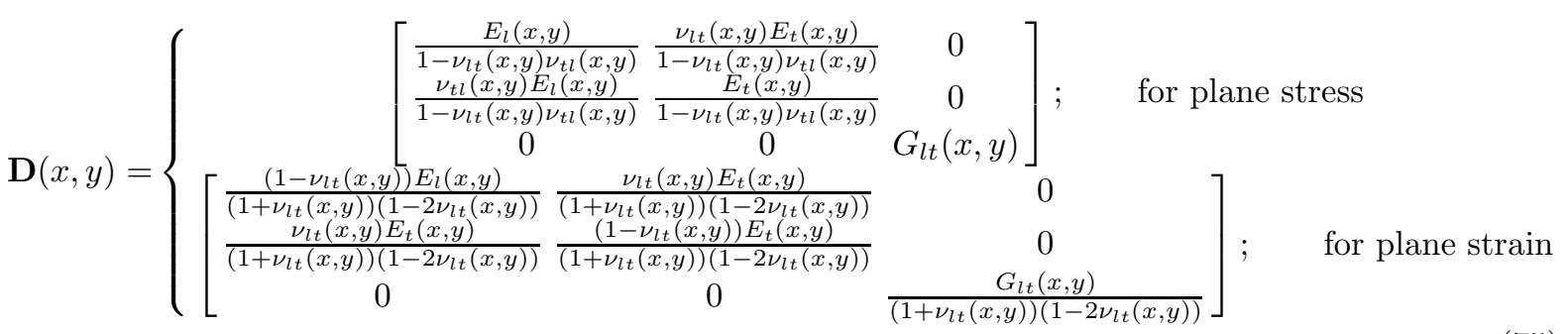

where $E_{l}(x, y)$ is the Young's modulus in the longitudinal direction, $E_{t}(x, y)$ is the Young's modulus in the transverse direction $G_{l t}(x, y)$ is the shear modulus; and $\nu_{l t}(x, y)$ and $\nu_{t l}(x, y)$ are the Poisson's ratio.

The material constitutive matrix is first expressed in terms of the scaled boundary coordinates $(\xi, \eta)$. The variation of the material properties $E(x, y), \nu(x, y), E_{l}(x, y)$, etc., over the two-dimensional space $(x, y)$ is assumed to be a polynomial function of the generic form

$$
\phi(x, y)=\phi_{0}+\phi_{1} x+\phi_{2} y+\phi_{3} x^{2}+\phi_{4} x y+\phi_{5} y^{2}+\ldots
$$

where $\phi$ can be substituted with the material parameters $E(x, y), \nu(x, y), E_{l}(x, y)$, etc. The constants $\phi_{i}$ can be obtained from a least squares fit in each polygon for a known material gradient. Using the scaled boundary transformation equation in Eq. (1), $\phi(x, y)$ can be expressed in scaled boundary coordinates $(\xi, \eta)$ as

$$
\phi(\xi, \eta)=\phi_{0}+\phi_{1} \xi x_{\eta}+\phi_{2} \xi y_{\eta}+\phi_{3} \xi^{2} x_{\eta}^{2}+\phi_{4} \xi^{2} x_{\eta} y_{\eta}+\phi_{5} \xi^{2} y_{\eta}^{2}+\ldots
$$

Collecting similar terms, $\phi(\xi, \eta)$ can be expressed as

$$
\begin{aligned}
\phi(\xi, \eta) & =\phi_{0}+\left(\phi_{1} x_{\eta}+\phi_{2} y_{\eta}\right) \xi+\left(\phi_{3} x_{\eta}^{2}+\phi_{4} x_{\eta} y_{\eta}+\phi_{5} y_{\eta}^{2}\right) \xi^{2}+\ldots \\
& =\sum_{r=0} \sum_{s=0}^{r} \xi^{r} \boldsymbol{\varphi}_{r s}(\eta)
\end{aligned}
$$

This representation allows the material constitutive matrix to be expressed as

$$
\mathbf{D}(\xi, \eta)=\sum_{r=0} \sum_{s=0}^{r} \xi^{r} \mathbf{D}_{r s}(\eta)
$$


The strain field $\varepsilon$ is first expressed in terms of scaled boundary coordinates $(\xi, \eta)$. Using Eq. (17) and Eq. (68) results in

$$
\varepsilon(\xi, \eta)=\boldsymbol{\Psi}_{\varepsilon}(\eta) \xi^{\mathbf{S}-\mathbf{I}} \mathbf{T u}_{0}
$$

where the strain mode $\boldsymbol{\Psi}_{\varepsilon}(\eta)$ is expressed as

$$
\boldsymbol{\Psi}_{\varepsilon}(\eta)=\mathbf{B}_{1}(\eta) \boldsymbol{\Psi}_{u} \mathbf{S}+\mathbf{B}_{2}(\eta) \boldsymbol{\Psi}_{u}
$$

Substituting Eq. (80) into the left-hand-side of Eq. (73) and using the Hooke's law with D defined by Eq. (79) results in

$$
\int_{\Omega} \delta \varepsilon^{T} \boldsymbol{\sigma} d \Omega=\delta \mathbf{u}_{0}^{T} \mathbf{T}^{T} \int_{\Omega} \xi^{\mathbf{S}^{T}-\mathbf{I}} \boldsymbol{\Psi}_{\varepsilon}^{T}(\eta)\left(\sum_{r=0} \sum_{s=0}^{r} \xi^{r} \mathbf{D}_{r s}(\eta)\right) \boldsymbol{\Psi}_{\varepsilon}(\eta) \xi^{\mathbf{S}-\mathbf{I}} d \Omega \mathbf{T u}_{0}
$$

The stiffness matrix is defined in the above equation by

$$
\mathbf{K}_{p o l}=\mathbf{T}^{T} \int_{\Omega} \xi^{\mathbf{S}^{T}-\mathbf{I}} \boldsymbol{\Psi}_{\epsilon}^{T}(\eta)\left(\sum_{r=0} \sum_{s=0}^{r} \xi^{r} \mathbf{D}_{r s}(\eta)\right) \boldsymbol{\Psi}_{\epsilon}(\eta) \xi^{\mathbf{S}-\mathbf{I}} d \Omega
$$

Substituting Eq. (7) for $d \Omega, \mathbf{K}_{\text {pol }}$ can be further expressed as

$$
\mathbf{K}_{p o l}=\mathbf{T}^{T} \sum_{r=0} \int_{0}^{1} \xi^{\mathbf{S}^{T}+(r-1) \mathbf{I}} \mathbf{Y}^{(r)}(\eta) \xi^{\mathbf{S}} d \xi \mathbf{T}
$$

where for each term $r$, the matrix $\mathbf{Y}^{(r)}(\eta)$ is

$$
\mathbf{Y}^{(r)}(\eta)=\sum_{s=0}^{r} \int_{-1}^{1} \boldsymbol{\Psi}_{\epsilon}^{T}(\eta) \mathbf{D}_{r s}(\eta) \boldsymbol{\Psi}_{\epsilon}(\eta) J(\eta) d \eta
$$

$\mathbf{Y}^{(r)}(\eta)$ can be integrated numerically using standard gaussian quadrature.

The stiffness matrix is evaluated as the sum of each term $r$. Defining the matrix

$$
\mathbf{X}^{(r)}=\int_{0}^{1} \xi^{\mathbf{S}^{T}+(r-1) \mathbf{I}} \mathbf{Y}^{(r)} \xi^{\mathbf{S}} d \xi
$$

for each term $r$ enables the stiffness matrix to be expressed as

$$
\mathbf{K}_{p o l}=\sum_{r=0} \mathbf{T}^{T} \mathbf{X}^{(r)} \mathbf{T}
$$

The matrix $\mathbf{X}^{(r)}$ is determined by integrating Eq. (86) by parts. This results in the Lyapunov equation

$$
(\mathbf{S}+0.5 r \mathbf{I})^{T} \mathbf{X}^{(r)}+\mathbf{X}^{(r)}(\mathbf{S}+0.5 r \mathbf{I})=\mathbf{Y}^{(r)}
$$

$\mathbf{X}^{(r)}$ is the solution of the Lyapunov equation in Eq. (88).

\subsection{Polygon load vector}

The polygon load vector $\mathbf{F}_{p o l}$ is defined in the external work component on the right-hand-side of Eq. (73)

$$
W_{e x t}=\int_{\Omega} \delta \mathbf{u}^{T} \mathbf{b} d \Omega+\int_{\Gamma} \delta \mathbf{u}^{T} \mathbf{t} d \Gamma
$$

Substituting for $d \Omega$ and $d \Gamma$ with Eq. (7) and Eq. (8), respectively and substituting for $\mathbf{u}(\xi, \eta)$ using Eq. (68)

$$
W_{e x t}=\delta \mathbf{u}_{0}^{T}\left(\mathbf{T}^{T} \int_{0}^{1} \xi^{\mathbf{S}+\mathbf{I}} \int_{-1}^{1} \boldsymbol{\Psi}_{u}^{T} \mathbf{N}(\eta) \mathbf{b} J d \eta d \xi+\left.\mathbf{T}^{T} \xi^{\mathbf{S}+\mathbf{I}} \int_{-1}^{1} \boldsymbol{\Psi}_{u}^{T} \mathbf{N}(\eta) \mathbf{t}(\eta) \Delta_{\Gamma} d \eta\right|_{\xi=1}\right)
$$


The load vector due to the body forces $\mathbf{F}_{\text {bod }}$ and the load vector due to the surface traction $\mathbf{F}_{\text {tra }}$ are defined in Eq. (90) by

$$
\begin{aligned}
& \mathbf{F}_{\text {bod }}=\mathbf{T}^{T} \int_{0}^{1} \xi^{\mathbf{S}+\mathbf{I}} \int_{-1}^{1} \boldsymbol{\Psi}_{u}^{T} \mathbf{N}(\eta) \mathbf{b} J d \eta d \xi \\
& \mathbf{F}_{\text {tra }}=\left.\mathbf{T}^{T} \xi^{\mathbf{S}+\mathbf{I}} \int_{-1}^{1} \boldsymbol{\Psi}_{u}^{T} \mathbf{N}(\eta) \mathbf{t}(\eta) \Delta_{\Gamma} d \eta\right|_{\xi=1}
\end{aligned}
$$

The distributed load vector due to surface tractions is evaluated only on the polygon boundar $(\xi=1)$ resulting in

$$
\mathbf{F}_{t r a}=\mathbf{T}^{T} \boldsymbol{\Psi}_{u}^{T} \int_{-1}^{1} \mathbf{N}^{T}(\eta) \mathbf{t}(\eta) \Delta_{\Gamma} d \eta
$$

In Eq. (93), note that

$$
\mathbf{T}^{T} \boldsymbol{\Psi}_{u}^{T}=\left[\begin{array}{ll}
\mathbf{I} & \\
& \mathbf{0}
\end{array}\right]
$$

Therefore, only the integral on the boundary contribute to the distributed load. This integral can be evaluated using standard Gaussian quadrature.

The body load distribution $\mathbf{b}$ in Eq. (91) is assumed to be in the form of a power function in scaled boundary coordinates $(\xi, \eta)$

$$
\mathbf{b}(\xi, \eta)=\sum_{j=0} \mathbf{b}^{(j)}(\eta) \xi^{j}
$$

Substituting Eq. (95) into Eq. (91) results in

$$
\mathbf{F}_{b o d}=\mathbf{T}^{T} \int_{0}^{1} \xi^{\mathbf{S}^{T}+\mathbf{I}} \boldsymbol{\Psi}_{u}^{T} \int_{-1}^{1} \mathbf{N}^{T}(\eta)\left(\sum_{j=0} \mathbf{b}^{(j)}(\eta) \xi^{j}\right) d \eta d \xi
$$

For a typical term with index $j$, the body force vector is $\mathbf{F}_{b o d}^{(j)}$ is

$$
\mathbf{F}_{b o d}^{(j)}=\mathbf{T}^{T} \int_{0}^{1} \xi^{\mathbf{S}^{T}+(j+1) \mathbf{I}} \boldsymbol{\Psi}_{u}^{T} \int_{-1}^{1} \mathbf{N}^{T}(\eta) \mathbf{b}^{(j)}(\eta) d \eta d \xi
$$

Integrating analytically results in

$$
\mathbf{F}_{b o d}^{(j)}=\mathbf{T}^{T}\left(\overline{\mathbf{S}}^{T}+(j+2) \mathbf{I}\right)^{-1} \boldsymbol{\Psi}_{u}^{T} \int_{-1}^{1} \mathbf{N}^{T}(\eta) \mathbf{b}^{(j)}(\eta) d \eta
$$

Considering all the terms, the total body force vector can be expressed as

$$
\mathbf{F}_{b o d}=\sum_{j=0}\left(\mathbf{T}^{T}\left(\overline{\mathbf{S}}^{T}+(j+2) \mathbf{I}\right)^{-1} \boldsymbol{\Psi}_{u}^{T} \int_{-1}^{1} \mathbf{N}^{T}(\eta) \mathbf{b}^{(j)}(\eta) d \eta\right)
$$

The total load vector in a polygon is therefore

$$
\mathbf{F}_{\text {pol }}=\mathbf{F}_{\text {bod }}+\mathbf{F}_{\text {tra }}
$$




\section{Numerical examples}

In this section, several numerical benchmarks are used to validate the developed formulation. Where applicable, the computed results are compared with those obtained using the analytical solution, the isoparametric graded finite element (Kim and Paulino, 2002) and the original SBFEM formulation for FGMs (Chiong et al, 2014).

In the analyses that follow, the number of required body force modes required for the bubble functions $\boldsymbol{\Phi}^{(b)}(\xi, \eta)$ for a specific order of shape function $\boldsymbol{\Phi}(\xi, \eta)$, is determined from the princples advocated by (Ooi et al, 2016). For second order shape functions $O(\eta)=2$, only a single body force mode is necessary. Higher order shape functions with $O(\eta)=3, O(\eta)=4$ and $O(\eta)=5$ require 3,6 and 10 body force modes in $\boldsymbol{\Phi}^{(b)}(\xi, \eta)$, respectively.

The order of the least squares fit required to determine the constants $\phi_{i}$ in Eq. (76) is determined by the order of the shape functions used to discretize the polygon boundary. For the simulations reported in this manuscript, the order of the least squares fit required to compute the constants $\phi_{i}$ is equal to the order of shape functions $O(\eta)$ used to discretize the polygon boundary. For problems where the gradient is a function of only one of the Cartesian axes, $x$ or $y$, the material properties are sampled at the integration points at $\xi=1$. For problems where the gradient is a function both $x$ and $y$, the material properties are sampled at the integration points at $\xi=1$ and $\xi=0.5$.

\subsection{Functionally graded plate under different loading conditions}

The functionally graded plate of infinite length $(L \rightarrow \infty)$ under plane stress conditions shown in Fig. 2a is considered. The width of the plate is $W$. Two loading cases are considered: (i) tension loading as is shown in Fig. 2b and; (ii) bending loading as is shown in Fig. 2c.

To simulate the infinite length of the plate, the computational domain is constructed with $\frac{L}{W}=4$. Two types of meshes are considered: (i) regular $N \times 4 N$ quadrilateral mesh; and (ii) arbitrary sided polygon mesh. Fig. 3 shows the generic meshes used in the simulations. Unless otherwise stated, $N$ is chosen to be $N=3$ and $N=9$ for regular quadrilateral meshes. The polygon meshes are generated from a Delaunay triangulated mesh following the approach of Ooi et al (2012). The mesh density is controlled by the size of the triangular element mesh $h_{t r i}$. For this simulation, $h_{t r i}$ was chosen to be $h_{t r i}=4.5$ and $h_{t r i}=1.125$.

The analytical solution of the plate under different types of loading conditions (tensile, bending and fixed gripped) for exponential and linear material variation were reported by Kim and Paulino (2002). These solutions will be used to as a reference to validate the developed formulation.

\subsubsection{Isotropic functionally graded plate}

The case of an isotropic functionally graded plate subjected to tensile loading is examined first. Two types of material gradient are considered: (i) material gradient perpendicular to the loading direction and; (ii) material gradient parallel to the loading direction. For the case where the material gradient is perpendicular to the loading direction, two types of material gradients are investigated; exponential and linear. For the material gradient that varies exponentially, the Poisson's ratio is constant and the Young's modulus $E$ varies exponentially as

$$
E(x)=E(0) e^{\frac{x}{W} \ln \left(\frac{E(W)}{E(0)}\right)}
$$

For the case of linear material variation, the Poisson's ratio $\nu$ is constant and

$$
E(x)=E_{0}+\frac{E(W)-E(0)}{W} x
$$

In both types of material gradients, the simulations are carried out with $E_{0}=E(0)=1 ; E(W)=8$ and $W=9$.

The simulations are performed using original SBFEM formulation, the SBFEM formulation with bubble functions, the isoparametric graded finite element formulation of Kim and Paulino (2002). The computed nodal displacements of each respective formulation are compared with the analytical solution. Quadratic (second order) shape functions are used for all formulations. 
Fig. 4a -4d show the error in the nodal displacements computed from the regular quadrilateral meshes and polygonal meshes for the case of exponential material gradient. For the case of linear material gradient, the error in the computed nodal displacements are shown in Fig. 5a - Fig. 5d. For these cases (loading and material gradient), the accuracy of all the formulations (isoparametric graded, SBFEM and SBFEM with bubble functions) are comparable and very close to the analytical solution.

The case where the material gradient is parallel to the loading direction is considered next. In this case, the Poisson's ratio $\nu=0.0$ and is constant over the domain. The Young's modulus varies according to

$$
E(y)=(1+y)^{n}
$$

Two cases are modelled: $n=1$ and $n=2$. The comparison of the accuracy of the computed nodal displacements with the analytical solutions for the case of $n=1$ are shown in Fig. 6a - Fig. 6d. For the case of $n=2$, the error in the computed nodal displacements are shown in Fig. 7a - Fig. 7d . For these cases, the accuracy of the SBFEM with bubble functions and the graded finite element formulation of Kim and Paulino (2002) are comparable when quadratic shape functions are used. Higher accuracy can be achieved by increasing the order of the bubble functions used.

A distinction in the solution accuracy can be observed when comparing the performance of the original SBFEM for cases where the material gradient is perpendicular; and parallel to the loading direction. In the former case, the accuracy of the SBFEM is comparable with the SBFEM with bubble functions. In the latter, the SBFEM with bubble functions show superior accuracy. This observation can be attributed to the nature of the analytical solution and also the high order completeness characteristic of the shape functions in the original SBFEM formulation. For the case where the material gradient is perpendicular to the direction of the load, the analytical displacement field is a polynomial function. It is quadratic in $u$ and linear in $v$ (Kim and Paulino, 2002). The SBFEM shape functions $\boldsymbol{\Phi}(\xi, \eta)$ are capable of reproducing all the terms in a linear polynomial unconditionally and all the terms in a quadratic polynomial under certain conditions. In the case where the material gradient is parallel to the direction of loading, the analyitical solution is a logarithmic function for $n=1$ and a rational function for $n=2$. The addition of the bubble functions $\boldsymbol{\Phi}^{(b)}(\xi, \eta)$ renders the SBFEM formulation to be high order complete (Ooi et al, 2016)and possess higher approximation capability. Therefore, its accuracy is superior compared with the SBFEM formulation without bubble functions.

The case of a isotropic functionally graded plate subjected to bending loading is now examined. The bending load $\sigma_{b}(x)$ in Fig. $2 \mathrm{c}$ is distributed as

$$
\sigma_{b}(x)=1-\frac{2}{9} x
$$

The Young's modulus $E(x)$ is assumed to vary exponentially according to Eq. (101) and the Poisson's ratio is assumed to be a constant i.e., $\nu(x)=0.3$. The error in the computed horizontal and vertical displacements computed using various element formulations are show in Fig. 8a - Fig. 8b in the case of quadrilateral meshes and Fig. 8c - Fig. 8d for polygonal meshes. For this particular case, just as in the case of uniform tensile load with material gradient perpendicular to the loading direction, the accuracy of the SBFEM formulation, the SBFEM formulation with bubble functions and the isoparametric graded elements are comparable and very close to the analytical solution.

\subsubsection{Orthotropic functionally graded plate}

The case of a functionally graded plate with orthotropic material properties subjected to tensile load $\sigma_{t}$ is now considered. The material gradients are perpendicular to the loading direction and vary exponentially with $x$ following

$$
\begin{aligned}
E_{l}(x) & =E_{l}(0) e^{\frac{x}{W} \ln \left(\frac{E_{l}(W)}{E_{l}(0)}\right)} \\
E_{t}(x) & =E_{t}(0) e^{\frac{x}{W} \ln \left(\frac{E_{t}(W)}{E_{t}(0)}\right)} \\
G_{l t}(x) & =G_{l t}(0) e^{\frac{x}{W} \ln \left(\frac{G_{l t}(W)}{G_{l t}(0)}\right)} \\
\nu_{l t}(x) & =0.3
\end{aligned}
$$

The simulations are carried out with material parameters $E_{l}(0)=1 ; E_{t}(0)=0.1$ and $G_{l t}(0)=0.5$. The error in the computed nodal displacements using the SBFEM formulation, the SBFEM formulation with 
bubble functions and the isoparametric graded elements are shown in Fig. 9a - Fig. 9d. For the quadrilateral meshes, the accuracy of all the three formulations (SBFEM, SBFEM with bubble functions and isoparametric graded) are comparable. They are very close to the analytical solution. For the polygonal meshes, the SBFEM formulation with bubble functions is more accurate. This may be attributed its the high order completeness property, which improves the approximation capability of the shape functions.

4.2 Rate of convergence for Poisson problem

A square domain $\Omega \in[0,1]^{2}$ is considered the Lame constants $\mu(x)$ and $\lambda(x)$ are assumed to vary according to

$$
\begin{aligned}
& \lambda(x)=\frac{E(x) \nu}{(1+\nu)(1+2 \nu)} \\
& \mu(x)=\frac{E(x)}{2(1+\nu)}
\end{aligned}
$$

where the Young's modulus $E(x)$ is defined as in Eq. (101) with $E_{0}=1$ and the Poisson's ratio $\nu$ is constant. The equilibrium condition of the domain subjected to body loads $b_{x}$ and $b_{y}$ is described by the Navier equations of elasticity

$$
\begin{array}{r}
(\lambda(x)+\mu(x))\left(\frac{\partial^{2} u}{\partial x^{2}}+\frac{\partial^{2} v}{\partial x \partial y}\right)+\mu(x)\left(\frac{\partial^{2} u}{\partial x^{2}}+\frac{\partial^{2} u}{\partial y^{2}}\right)+\frac{\partial \lambda(x)}{\partial x}\left(\frac{\partial u}{\partial x}+\frac{\partial v}{\partial y}\right)+2 \frac{\partial \mu(x)}{\partial x} \frac{\partial u}{\partial x}+b_{x}=0 \\
(\lambda(x)+\mu(x))\left(\frac{\partial^{2} v}{\partial y^{2}}+\frac{\partial^{2} u}{\partial x \partial y}\right)+\mu(x)\left(\frac{\partial^{2} v}{\partial y^{2}}+\frac{\partial^{2} v}{\partial x^{2}}\right)+\frac{\partial \mu(x)}{\partial x}\left(\frac{\partial u}{\partial y}+\frac{\partial v}{\partial x}\right)+b_{y}=0
\end{array}
$$

The domain is discretized using two approaches: (i) $N \times N$ structured quadrilateral meshes and (ii) arbitrary sided polygon meshes. The quadrilateral meshes are generated using $N=2, N=4, N=8$ and $N=16$. For the polygon meshes, the generic size of a polygon is determined by the parameter $h_{t r i}$. The polygon meshes are generated using $h_{t r i}=1 / M$. The values of $N$ used are $M=4, M=8, M=16$ and $M=32$.

A convergence study is carried out for both mesh types; quadrilaterals and polygons. The results obtained from the SBFEM formulation with bubble functions are compared with the original SBFEM formulation developed for functionally graded materials (Chiong et al, 2014). The $L_{2}-$ norm of the relative error in the displacements is monitored. The norm is defined according to Talischi and Paulino (2014); Talischi et al (2015) as

$$
\left\|\mathbf{u}_{h}-\mathbf{u}\right\|_{2}=\sqrt{\int_{\Omega}\left(\mathbf{u}_{h}-\mathbf{u}\right)^{T}\left(\mathbf{u}_{h}-\mathbf{u}\right) d \Omega}
$$

where $\mathbf{u}_{h}$ is the computed displacement vector and $\mathbf{u}$ is the analytical displacement vector. Eq. (113) can be integrated numerically.

The case of a polynomial displacement field of the form

$$
u(x, y)=v(x, y)=x^{2} y^{2}(1-x)(1-y)
$$

is considered first. The body force intensities for this problem are derived from Eq. (111) and Eq. (112) and the material gradient. Fig. 11a and Fig. 11b compare the convergence of the formulations for the quadrilateral and the polygonal meshes, respectively. A similar trend is observed for both types of meshes considered. The addition of the bubble functions improves both the accuracy and the convergence rate of the SBFEM. For the regular quadrilateral mesh with quadratic shape functions $(O(\eta)=2)$, the convergence rate for the SBFEM is 1.065 compared with 1.617 when the bubble functions are used. The convergence rate for the bubble functions can be increased further when higher order shape functions are used. For the third and fourth order shape functions, $O(\eta)=3$ and $O(\eta)=4$, the convergence rates are 2.091 and 2.225, respectively. When the polygonal meshes are used, the convergence rate is 1.038 for the SBFEM with quadratic shape functions compared with 1.607 when bubble functions are used. The convergence rates are 2.107 and 2.236 when the third and fourth order shape functions are used. 
The case of a trigonometric displacement field of the form

$$
u(x, y)=v(x, y)=x y(1-x)(1-y) \sin (3 \pi x) \cos (2 \pi y)
$$

is considered next. The convergence study is repeated using the same meshes used in the case of a polynomial displacement field. The results elucidating the performances of the SBFEM and the use of bubble functions are shown in Fig. 12. Similar to the case where a polynomial displacement field is considered, the inclusion of bubble functions demonstrate increased convergence rates. For the regular quadrilateral mesh, comparing shape functions of $O(\eta)=2$, the convergence rate is 0.9911 with the original SBFEM and this improves to 1.571 when the bubble functions are used. For the high order bubble functions, the convergence rates are 2.152 when the shape functions are of order $O(\eta)=3$ and 2.614 when the shape functions are of order $O(\eta)=4$. For the polygonal meshes, the convergence rates with shape functions of order $O(\eta)=2$ are 1.157 and 1.707 for the original SBFEM formulation and the SBFEM formulation with bubble functions, respectively. The convergence rates are further improved when higher order shape functions are used. For shape functions of order $O(\eta)=3$ and $O(\eta)=4$, the convergence rates are 2.355 and 2.637, respectively. Unlike the case where the displacement field is a pure polynomial expression, the accuracy of the coarse meshes using higher order shape functions is lower compared with a fine mesh using a lower order shape function with the same number of degreesof-freedom. However, the accuracy rapidly increases with increase in mesh density and this is reflected by the high convergence rates associated with the high order shape functions.

Just like the displacements, the strains $\boldsymbol{\epsilon}=\left[\begin{array}{lll}\epsilon_{x x} & \epsilon_{y y} & \gamma_{x y}\end{array}\right]$ can be computed accurately within the domain $\Omega$ when the bubble functions are incorporated into the SBFEM formulation. Fig. 13 shows the contour plots of the error in the computed strain components for the regular quadrilateral and arbitrary polygonal meshes using bubble functions of the order $O(\eta)=3$. It can be identified from the Fig. 13 that the strain components can be accurately reproduced within the domain.

\subsection{Infinite functionally graded plate with a circular hole}

The infinite functionally graded plate with a circular hole subjected to uniform bi-axial tension $T$ is considered. due to symmetric conditions, only quarter of the plate is modelled. The geometry and boundary conditions pertinent to the plate are shown in Fig. 14a. The Poisson's ratio $\nu=0.25$ and is constant over the entire plate. The Young's modulus is graded radially according to

$$
E(r)=E_{0}\left(\frac{r}{a}\right)^{n}
$$

where

$$
r=\sqrt{x^{2}+y^{2}}
$$

The analytical solution for this problem derived by Saddd (2005) from a hollow cylindrical domain subjected to uniform internal and external pressure was validated using the FEM by Berlo (2009). This problem will be used as another numerical benchmark to validate the SBFEM formulation with bubble functions. Two polygonal meshes shown in Fig. 14b and Fig. 14c are considered. The coarse mesh comprise of 89 polygons. The fine mesh has 217 polygons.

The elastic analysis was carried out with the following material and geometric parameters; $E_{0}=1$, $a=1, b=20$. The effects of the material gradient on the stress distribution is investigated for two values of $n ; n=0.2$ and $n=-0.2$. Plane stress conditions are assumed. Shape functions of order $O(\eta)=2$ and $O(\eta)=3$ are used in the analyses. For the $O(\eta)=2$ shape functions, the mesh has 498 nodes in the coarse mesh and 1186 nodes in the fine mesh. For the $O(\eta)=3$ shape functions, the mesh has 791 nodes in the course mesh and 1887 nodes in the fine mesh.

Fig. 15a and Fig. 15b compares the distribution of the normalized circumferential stress $\sigma_{\theta \theta} / T$ with respect to the normalized distance $r / a$ obtained from the developed formulation with the available analytical solution. It is evident from Fig. 15a and Fig. 15b that the developed method can reproduce the correct stress distribution with sufficient accuracy in both coarse and fine meshes irrespective of the order of the shape functions used. Table 1 compares the predicted maximum cirumferential stress for the different material gradients considered using the second and third order shape functions. It can be clearly identified in Table 1 that the predicted stresses are very close to the analytical solution. The error in the SBFEM solutions were less than $0.5 \%$. 
Table 1: Comparison of predicted maximum circumferential stress in the infinite functionally graded plate with a circular hole problem.

\begin{tabular}{lcccc}
\hline & & \multicolumn{3}{c}{$\sigma_{\theta \theta} / T$} \\
\hline \hline \multirow{2}{*}{$n=0.2$} & coarse & $1.5430=2$ & $O(\eta)=3$ & Analytical \\
& fine & 1.5465 & 1.5477 & \multirow{2}{*}{1.5473} \\
\multirow{2}{*}{$n=-0.2$} & coarse & 2.5344 & 2.5613 & \multirow{2}{*}{2.5482} \\
& fine & 2.5423 & 2.5560 & \\
\hline
\end{tabular}

Fig. 16a and Fig. 16b show the contour plot of the normalized circumferential stress $\sigma_{\theta \theta} / T$ for the case where $n=0.2$ and $n=-0.2$. The radial distribution of $\sigma_{\theta \theta} / T$, which is characteristic to the analytical solution is clearly evident from the contour plots in the vicinity of the hole.

4.4 Stress intensity factors in a functionally graded medium subjected to body load

A functionally graded region defined by $\Omega \in[-5,5]^{2}$ with a central crack of length $a=1$ shown in Fig. 17 is considered. The boundary of the plate is constrained from motion in all directions. The plate is subjected to a body load intensity of the form

$$
\left\{\begin{array}{l}
b_{x} \\
b_{y}
\end{array}\right\}=\left\{\begin{array}{l}
0 \\
y
\end{array}\right\} \times 10^{5}
$$

The Young's modulus of the plate is graded according to

$$
E(y)=(-4 y+40) \times 10^{9}
$$

The Poisson's ratio $\nu=0.3$ and is assumed to be constant. Plane strain conditions are assumed. The stress intensity factors of the crack in the plate are computed considering crack angles $\theta=0^{\circ}, \theta=30^{\circ}$ and $\theta=60^{\circ}$.

The plate is discretised using arbitrary sided polygons. The mesh density is characterized by a parameter $h$, representing approximately the average circumscribed radii of the polygons in the mesh. Fig. 18 shows two typical meshes that are used to discretize the plate for the case where $\theta=0^{\circ}$. The convergence behaviour of the SBFEM formulation with bubble functions is investigated by: (i) increasing the mesh density by reducing the parameter $h$ and; (ii) increasing the order of the shape functions used in the polygons. The computed stress intensity factors are compared with those obtained from the SBFEM formulation without bubble functions.

When the bubble functions are used, the procedure to compute the stress intensity factors do not differ significantly from that of the SBFEM without bubble functions. In this manuscript, the stress intensity factors are computed using the solutions of the displacements. This approach is adapted from Chiong et al (2014). The stress intensity factors are evaluated as

$$
\left\{\begin{array}{c}
K_{I} \\
K_{I I}
\end{array}\right\}=\frac{G_{t i p}}{\kappa_{t i p}+1} \sqrt{\frac{2 \pi}{r_{0}}}\left\{\begin{array}{c}
u_{\theta A}^{(s)}-u_{\theta B}^{(s)} \\
u_{r A}^{(s)}-u_{r B}^{(s)}
\end{array}\right\}
$$

where $r_{0}$ is the crack length measured from the crack tip to the crack mouth in the crack polygon, $G_{t i p}$ is the shear modulus at the crack tip, $\kappa_{t i p}$ is evaluated as

$$
\kappa_{\text {tip }}= \begin{cases}3-4 \nu_{\text {tip }} & \text { plane strain } \\ \left(3-4 \nu_{\text {tip }}\right) /\left(1+4 \nu_{\text {tip }}\right) & \text { plane stress }\end{cases}
$$

with $\nu_{t i p}$ representing the Poisson's ratio at the crack tip. The nodal displacement vectors in radial coordinates, $\left[\begin{array}{ll}u_{\theta A}^{(s)} & u_{r A}^{(s)}\end{array}\right]$ and $\left[\begin{array}{ll}u_{\theta B}^{(s)} & u_{r B}^{(s)}\end{array}\right]$, are computed from Eq. (55) considering only the displacement modes corresponding to the diagonal block in $\mathbf{S}$ with eigenvalues $\lambda$ satisfying $-1 \leq \lambda \leq 0$. This diagonal block leads to singular stresses when $\xi \rightarrow 0$. Since the eigenvalues due to the body force modes in $\boldsymbol{\Lambda}_{b}$ always satisfy $\lambda\left(\boldsymbol{\Lambda}_{b}\right)>0$, the second term in Eq. (55) does not contribute to the stress intensity factors. The $2 \times 2$ diagonal block that leads to singular stresses when $\xi \rightarrow 0, \mathbf{S}^{(s)}$, is therefore, defined only in $\boldsymbol{\Lambda}_{n}$. The Cartesian nodal displacment vector that lead to singular stresses as $\xi \rightarrow 0, \mathbf{u}^{(s)}$, is then computed as

$$
\mathbf{u}^{(s)}=\mathbf{V}_{n}^{(s)} \mathbf{c}^{(s)}
$$


where $\mathbf{V}_{n}^{(s)}$ is an $N \times 2$ matrix corresponding to the diagonal block $\mathbf{S}^{(s)}$ and $\mathbf{c}^{(s)}$ are the integration constants corresponding to $\mathbf{S}^{(s)}$. The nodal displacements at $A$ and $B$ can be extracted from $\mathbf{u}^{(s)}$ and can be transformed into polar coordinates using standard procedures to used in Eq. (120).

The convergence of the stress intensity factors with increasing mesh density is first discussed. The stress intensity factors are normalized by as $K_{I}^{n o r m}=K_{I} / \sqrt{\pi a} \times 10^{-6}$ and $K_{I I}^{n o r m}=K_{I I} / \sqrt{\pi a} \times 10^{-6}$. In this investigation, third order shape functions are used. Tables $2 \mathrm{a}-\mathrm{c}$ show the normalized stress intensity factors for $\theta=0^{\circ}, \theta=30^{\circ}$ and $\theta=60^{\circ}$. The results in Tablea 2a-c indicate that the normailsed stress intensity factors computed with the bubble functions converge more rapidly compared with the stress intensity factors computed with the SBFEM alone. This applies to all the crack angles that are considered. The stress intensity factors computed with the SBFEM appear to be converging towards the value of the stress intensity factors computed with the bubble functions when $h=0.25$. This observation indicates that the bubble functions improve the accuracy of the SBFEM for fracture problems in the presence of body loads. The increased in accuracy gained is through the more accurate integration constants that include contributions from the bubble functions.

Table 2: Convergence of stress intensity factors with increasing mesh density.

\begin{tabular}{|c|c|c|c|c|c|c|c|c|}
\hline \multicolumn{9}{|c|}{ Normalised Stress Intensity Factors } \\
\hline & \multicolumn{2}{|c|}{$K_{I}^{(\text {left } t)}$} & \multicolumn{2}{|c|}{$\bar{K}_{I}^{(\text {right })}$} & \multicolumn{2}{|c|}{$K_{U}^{(\text {left })}$} & \multicolumn{2}{|c|}{$K_{L}^{(\text {right })}$} \\
\hline & SBFEM & Bubble & SBFEM & Bubble & SBFEM & Bubble & SBFEM & Bubble \\
\hline$h=4.0$ & 0.416097 & 0.425907 & 0.416097 & 0.4259077 & 0.000000 & 0.000000 & 0.000000 & 0.000000 \\
\hline$h=2.0$ & 0.422745 & 0.426054 & 0.422055 & 0.425899 & 0.000000 & 0.000000 & 0.000000 & 0.000000 \\
\hline$h=1.0$ & 0.423979 & 0.425987 & 0.423961 & 0.425984 & 0.000000 & 0.000000 & 0.000000 & 0.000000 \\
\hline$h=0.5$ & 0.425467 & 0.425967 & 0.425467 & 0.425967 & 0.000000 & 0.000000 & 0.000000 & 0.000000 \\
\hline$h=0.25$ & 0.425846 & 0.425956 & 0.425848 & 0.425957 & 0.000000 & 0.000000 & 0.000000 & 0.000000 \\
\hline
\end{tabular}

(a) $\theta=0^{\circ}$

\begin{tabular}{|c|c|c|c|c|c|c|c|c|}
\hline \multicolumn{9}{|c|}{ Normalised Stress Intensity Factors } \\
\hline & \multicolumn{2}{|c|}{$K_{I}^{(\text {left })}$} & \multicolumn{2}{|c|}{$K_{I}^{(\text {right })}$} & \multicolumn{2}{|c|}{$K_{I I}^{(\operatorname{left} t)}$} & \multicolumn{2}{|c|}{$K_{I I}^{(\text {right })}$} \\
\hline & SBFEM & Bubble & SBFEM & Bubble & SBFEM & Bubble & SBFEM & Bubble \\
\hline$h=4.0$ & 0.332919 & 0.349165 & 0.333688 & 0.349672 & 0.121431 & 0.135232 & 0.1085839 & 0.115211 \\
\hline$h=2.0$ & 0.342895 & 0.349084 & 0.342071 & 0.349809 & 0.130654 & 0.135430 & 0.1126616 & 0.115042 \\
\hline$h=1.0$ & 0.347102 & 0.348888 & 0.347657 & 0.350242 & 0.133981 & 0.135474 & 0.1140066 & 0.114715 \\
\hline$h=0.5$ & 0.348195 & 0.348518 & 0.349948 & 0.350621 & 0.134989 & 0.135434 & 0.1145941 & 0.114728 \\
\hline$h=0.25$ & 0.348263 & 0.348304 & 0.350639 & 0.350841 & 0.135279 & 0.135423 & 0.1147059 & 0.114713 \\
\hline
\end{tabular}

(b) $\theta=30^{\circ}$

\begin{tabular}{|c|c|c|c|c|c|c|c|c|}
\hline \multicolumn{9}{|c|}{ Normalised Stress Intensity Factors } \\
\hline & \multicolumn{2}{|c|}{$K_{I}^{(l e f t)}$} & \multicolumn{2}{|c|}{$K_{I}^{(\text {right })}$} & \multicolumn{2}{|c|}{$K_{I I}^{(\text {left })}$} & \multicolumn{2}{|c|}{$K_{I I}^{(\text {right })}$} \\
\hline & SBFEM & Bubble & SBFEM & Bubble & SBFEM & Bubble & SBFEM & Bubble \\
\hline$h=4.0$ & 0.196399 & 0.202457 & 0.195213 & 0.202988 & 0.125180 & 0.126968 & 0.112403 & 0.115019 \\
\hline$h=2.0$ & 0.203170 & 0.203685 & 0.200631 & 0.203755 & 0.127451 & 0.127046 & 0.113468 & 0.114908 \\
\hline$h=1.0$ & 0.204743 & 0.203648 & 0.202405 & 0.203924 & 0.127300 & 0.127166 & 0.113809 & 0.114913 \\
\hline$h=0.5$ & 0.203109 & 0.203161 & 0.203983 & 0.204390 & 0.126393 & 0.126616 & 0.114967 & 0.115331 \\
\hline$h=0.25$ & 0.202992 & 0.202984 & 0.204435 & 0.204597 & 0.126384 & 0.126375 & 0.115466 & 0.115582 \\
\hline
\end{tabular}

(c) $\theta=60^{\circ}$

Next, the convergence of the computed stress intensity factors with increasing order of shape functions is discussed. For each crack angle $\theta$ considered, the polygon mesh with $h=0.5$ is used. The convergence of the computed stress intensity factors is monitored as the order of the shape functions $O(\eta)$ is increased. Tablea 3a-c show the computed normalized stress intensity factors. It is interesting to note here that for the SBFEM formulation, the normalized stress intensity factors is nearly constant and does not improve with when the order of the shape functions is increased. On the other hand, when the bubble functions are used, the computed stress intensity factors gradually improve when the order of the shape functions is increased. The values of the stress intensity factors approaches that obtained when $h=0.25$ shown in Tables 2a-c. This highlights the importance of the application of the bubble functions in problems involving body loads. 
Table 3: Convergence of stress intensity factors with increasing order of shape functions.

(a) $\theta=0^{\circ}$

\begin{tabular}{ccccccccc}
\hline \multicolumn{8}{c}{ Normalised Stress Intensity Factors } \\
\hline \hline \multicolumn{2}{c}{$K_{I}^{(\text {left })}$} & \multicolumn{2}{c}{$K_{I}^{\text {(right) }}$} & \multicolumn{2}{c}{$K_{I I}^{(\text {left })}$} & \multicolumn{2}{c}{$K_{I I}^{(\text {right })}$} \\
\hline$O(\eta)=2$ & SBFEM & Bubble & SBFEM & Bubble & SBFEM & Bubble & SBFEM & Bubble \\
\hline$(\eta)=3$ & 0.405983 & 0.406093 & 0.385176 & 0.385154 & 0.000000 & 0.000000 & 0.000000 & 0.000000 \\
$O(\eta)=4$ & 0.405943 & 0.405977 & 0.385128 & 0.385692 & 0.000000 & 0.000000 & 0.000000 & 0.000000 \\
$O(\eta)=5$ & 0.405943 & 0.405797 & 0.385126 & 0.385705 & 0.000000 & 0.000000 & 0.000000 & 0.000000 \\
$O(\eta)=6$ & 0.405943 & 0.405787 & 0.385127 & 0.385899 & 0.000000 & 0.000000 & 0.000000 & 0.000000 \\
$O(\eta)=7$ & 0.405943 & 0.405706 & 0.385127 & 0.385908 & 0.000000 & 0.000000 & 0.000000 & 0.000000 \\
$O(\eta)=2$ & 0.405943 & 0.405702 & 0.385127 & 0.386004 & 0.000000 & 0.000000 & 0.000000 & 0.000000 \\
\hline
\end{tabular}

(b) $\theta=30^{\circ}$

\begin{tabular}{|c|c|c|c|c|c|c|c|c|}
\hline \multicolumn{9}{|c|}{ Normalised Stress Intensity Factors } \\
\hline & \multicolumn{2}{|c|}{$K_{I}^{(l e f t)}$} & \multicolumn{2}{|c|}{$K_{I}^{(\text {right })}$} & \multicolumn{2}{|c|}{$K_{I I}^{(\text {left } t)}$} & \multicolumn{2}{|c|}{$K_{I}^{(\text {right })}$} \\
\hline & SBFEM & Bubble & SBFEM & Bubble & SBFEM & Bubble & SBFEM & Bubble \\
\hline$O(\eta)=2$ & 0.348227 & 0.348623 & 0.349988 & 0.350238 & 0.135006 & 0.135425 & 0.114608 & 0.114628 \\
\hline$O(\eta)=3$ & 0.348195 & 0.348518 & 0.349948 & 0.350621 & 0.134989 & 0.135434 & 0.114594 & 0.114728 \\
\hline$O(\eta)=4$ & 0.348193 & 0.348497 & 0.349946 & 0.350639 & 0.134988 & 0.135490 & 0.114593 & 0.114667 \\
\hline$O(\eta)=5$ & 0.348193 & 0.348380 & 0.349947 & 0.350761 & 0.134988 & 0.135459 & 0.114593 & 0.114685 \\
\hline$O(\eta)=6$ & 0.348193 & 0.348366 & 0.349947 & 0.350775 & 0.134988 & 0.135474 & 0.114593 & 0.114669 \\
\hline$O(\eta)=7$ & 0.348193 & 0.348310 & 0.349947 & 0.350833 & 0.134988 & 0.135453 & 0.114593 & 0.114683 \\
\hline$O(\eta)=2$ & 0.348193 & 0.348301 & 0.349947 & 0.350842 & 0.134988 & 0.135461 & 0.114593 & 0.114674 \\
\hline
\end{tabular}

(c) $\theta=60^{\circ}$

\begin{tabular}{|c|c|c|c|c|c|c|c|c|}
\hline \multicolumn{9}{|c|}{ Normalised Stress Intensity Factors } \\
\hline & \multicolumn{2}{|c|}{$K_{I}^{(l e f t)}$} & \multicolumn{2}{|c|}{ " $K_{I}^{(r i g h t)}$} & \multicolumn{2}{|c|}{$K_{I I}^{(l e f t)}$} & \multicolumn{2}{|c|}{$K_{I}^{(\text {right })}$} \\
\hline & SBFEM & Bubble & SBFEM & Bubble & SBFEM & Bubble & SBFEM & Bubble \\
\hline$\overline{O(\eta)}=2$ & 0.203124 & 0.203253 & $\overline{0.204004}$ & 0.204143 & 0.126401 & $\overline{0.126713}$ & 0.114976 & $\overline{0.115119}$ \\
\hline$O(\eta)=3$ & 0.203109 & 0.203161 & 0.203983 & 0.204390 & 0.126393 & 0.126616 & 0.114967 & 0.115331 \\
\hline$O(\eta)=4$ & 0.203108 & 0.203160 & 0.203983 & 0.204388 & 0.126392 & 0.126614 & 0.114966 & 0.115332 \\
\hline$O(\eta)=5$ & 0.203108 & 0.203058 & 0.203982 & 0.204507 & 0.126392 & 0.126487 & 0.114966 & 0.115465 \\
\hline$O(\eta)=6$ & 0.203108 & 0.203048 & 0.203982 & 0.204520 & 0.126392 & 0.126497 & 0.114966 & 0.115453 \\
\hline$O(\eta)=7$ & 0.203107 & 0.203005 & 0.203982 & 0.204570 & 0.126392 & 0.126407 & 0.114966 & 0.115548 \\
\hline$O(\eta)=2$ & 0.203107 & 0.202991 & 0.203982 & 0.204587 & 0.126392 & 0.126419 & 0.114966 & 0.115534 \\
\hline
\end{tabular}

\section{Conclusions}

The SBFEM formulation, which adopts high order complete bubble-like shape functions (Ooi et al, 2016) has been further developed for elasto-static analyses in FGMs. The same set of shape functions valid for homogeneous materials can be used. The heterogeneity resulting from the material gradient introduces additional terms in the polygon stiffness matrix that can be integrated analytically.

The developed formulation was successfully validated using four numerical benchmarks. It was found that:

1. The SBFEM formulation with bubble functions can reproduce the analytical solutions for all the numerical benchmarks considered. The accuracy of the solutions can be improved by either increasing the mesh density or by increasing the order of the one-dimensional shape functions used to discretize the polygon boundary.

2. Compared with the original SBFEM formulation, incorporating the bubble functions resulted in superior accuracy for problems where the analytical solution were not in polynomial forms. This was particularly evident in the case of isotropic FGMs where the material gradient was parallel to the direction of the load and in the case of orthotropic FGMs. The bubble functions also significantly improved the accuracy and convergence rate of the SBFEM for problems of the Poisson type.

3. For cracked bodies subjected to body loads, the bubble functions improved the accuracy of the computed stress intensity factors compared with using SBFEM alone. Increasing the order of the bubble functions improves the accuracy further. When the SBFEM is used without bubble functions, improvements in accuracy of the computed stress intensity factors can only be achieved by increasing the mesh density. 
4. The superior accuracy and convergence rate gained by introducing the bubble functions is attributed their high order completeness properties, which increased the interpolating capacity of the shape functions.

\section{References}

Bao G, Wang L (1995) Modelling cracking in functionally graded ceramic/metal coatings. International Journal of Solids and Structures 32:2853-2871

Behnke R, Mundil M, Birk C, Kaliske M (2014) A physically and geometrically nonlinear scaledboundary-based finite element formulation for fracture in elastomers. International Journal for $\mathrm{Nu}-$ merical Methods in Engineering 99:966-999

Belytschko T, Gu YY, Gu L (1994) Element-free galerkin methods. International Journal for Numerical Methods in Engineering 37:229-256

Berlo SP (2009) Stress concentration effects ih highly localized functionally graded materials. Masters thesis, University of Rhode Island

Birk C, Behnke R (2012) A modified scaled boundary finite element method for three-dimensional dynamic soil structure interaction in layered soil. International Journal for Numerical Methods in Engineering 89:371-402

Chen SS, Xu CJ, Tong GS (2015) A meshless local neighbour interpolation method to modeling of functionally graded viscoelastic materials. Engineering Analysis with Boundary Elements 52:92-98

Chiong I, Ooi ET, Song C, Tin-Loi F (2014) Scaled boundary polygons with application to fracture analysis of functionally graded materials. International Journal for Numerical Methods in Engineering 98:562-589

Enab TA (2014) Stress concentration analysis in functionally graded plates with elliptic holes under biaxial loadings. Ain Shams Engineering Journal 5:839-850

Gao XW (2002) A boundary element method with internal cells for two-dimensional and threedimensional elastoplastic problems. Journal of Applied Mechanics (ASME) 69:154-160

Goswami S, Becker W (2012) Computation of 3-d stress singlarities for multiple cracks and crack intersections by the scaled boundary finite element method. International Journal of Fracture 175:13-25

Hernik S (2010) New concept of finite element method for fgm materials. Czasopismo Techniczne Mechanika Politechniki Krakowskiej 107:99-106

Ibrahimbegovic A, Wilson EL (1991) A modified method of incompatible modes. Communications in Numerical Methods and Engineering 7:187-194

Kim JH, Paulino GH (2002) Isoparametric graded finite elements for nonhomogeneous isotropic and orthotropic materials. Journal of Applied Mechanics 69:502-514

Koizumi M (1997) Fgm activities in japan. Composites Part B: Engineering 28:1-4

Liu J, Peng HF, Gao XW, Cui M (2015) A traction-recovery method for evaluating boundary stresses on thermal elasticity problems of fgms. Engineering Analysis with Boundary Elements 61:226-231

Liu WK, Jun S, Zhang YF (1995) Reproducing kernel particle methods. International Journal for Numerical Methods in Fluids 20:1081-1106

Martinez-Paneda E, Gallego R (2015) Numerical analysis of quasi-static fracture in functionally graded materials. International Journal of Mechanical and Material Design 11:405-424

Meyer M, Barr A, Lee H, Desbrun M (2002) Generalized barycentric coordinates on irregular polygons. Journal of Graphic Tools 7:13-22

Natarajan S, Song C, Belouettar S (2014) Numerical evaluation of stress intensity factors and t-stress for interfacial cracks and cracks terminating at interface with asymptotic enrichment. Computer Methods in Applied Mechanics and Engineering 279:86-112

Nguyen VP, Rabczuk T, Bordas S, Dufolt M (2008) Meshless methods: A review and computer implementation aspects. Mathematics and Computers in Simulation 79(3):763-813

Ooi ET, Song C, Tin-Loi F, Yang ZJ (2012) Polygon scaled boundary finite elements for crack propagation modelling. International Journal for Numerical Methods in Engineering 91:319-342

Ooi ET, Song C, Tin-Loi F (2014) A scaled boundary polygon formulation for elasto-plastic analyses. Computer Methods in Applied Mechanics and Engineering 268:905-937

Ooi ET, Song C, Natarajan S (2016) Construction of high-order complete scaled boundary shape functions over arbitrary polygons with bubble functions. International Journal for Numerical Methods in Engineering 108:1086-1120 
Riveiro MA, Gallego R (2013) Boundary elements and the analog equation method for the solution of elastic problems in 3-d non-homogeneous bodies. Computer Methods in Applied Mechanics and Engineering 263:12-19

Rosseau CE, Tippur HV (2000) Compositionally graded materials with ccrack normal to the elastic gradient. Acta Materialia 48:4021-4033

Saddd MH (2005) Elasticity: Theory, Applications and Numerics. Elsevier Academic Press, ButterworthHeinemann

Santare AG, Lambros MH (2000) Use of graded finite elements to model the behaviour of nonhomogeneous materials. Journal of Applied Mechanics (ASME) 67:819-822

Sladek J, Sladek V, Zhang C (2005) Stress analysis in anisotropic functionally graded materials by the mlpg method. Engineering Analysis with Boundary Elements 29:597-609

Sladek J, Sladek V, Zhang C, Solek P, P E (2007) Evaluation of fracture parameters in continuously nonhomogeneous piezoelectroc solids. International Journal of Fracture 145:313-326

Sladek J, Sladek V, Zhang C (2008a) Evaluation of the stress intensity factors for cracks in continuously nonhomogeneous solids, part 1: Interaction integral. Mechanics of Advanced Materials and Structures 15:438-443

Sladek J, Sladek V, Zhang C (2008b) Evaluation of the stress intensity factors for cracks in continuously nonhomogeneous solids, part ii: Meshless method. Mechanics of Advanced Materials and Structures 15:444-452

Sladek J, Sladek V, Solek P, Zhang C (2010) Fracture analysis in continuously nonhomogeneous magnetoelectro-elastic solids under a thermal load by the mlpg. International Journal of Solids and Structures 47:1381-1391

Sladek J, Stanak P, Han ZD, Sladek V, Atluri SN (2013) Applications of the mlpg method in engineering and sciences: A review. Computer Modeling in Engineering and Sciences 92:423-475

Sladek V, Sladek J (1998) Singular integrals and boundary elements. Computer Methods in Applied Mechanics and Engineering 1998:251-266

Song C (2006) Analysis of singular stress fields at multi-material corners under thermal loading. International Journal for Numerical Methods in Engineering 65:620-652

Song C, Wolf JP (1997) The scaled boundary finite-element method-alias consistent infinitesimal finiteelement cell method-for elastodynamics. Computer Methods in Applied Mechanics and Engineering 147:329-355

Song H, Tao L (2010) An efficient scaled boundary fem model for wave interaction with a nonuniform porous cylinder. International Journal for Numerical Methods in Fluids 63:96-118

Sukumar N, Tabarraei A (2004) Conforming polygonal finite elements. International Journal for Numerical Methods in Engineering 61:2045-2066

Talischi C, Paulino GH (2014) Addressing integration error for polygonal finite elements trhough polynomial projections: A patch test connection. Mathematical Models and Methods in Applied Sciences 24:1701-1727

Talischi C, Pérereira A, Menezes IF, Paulino GH (2015) Gradient correction for polygonal and polyhedral finite elements. International Journal for Numerical Methods in Engineering 102:728-747

Taylor RL, Beresford PJ, Wilson EL (1976) A non-conforming element for stress analysis. International Journal for Numerical Methods in Engineering 10:1211-1219

Vatanabe SL, Paulino GH, Silva ECN (2013) Design of functionally graded piezocomposites using topology optimization and homogenization - toward effective energy harvesting materials. Computer Methods in Applied Mechanics and Engineering 266:205-218

Wachspress EL (1975) A Rational Finite Element Basis. Academic Press, New York

Wang H, Qin QH (2008) Meshless approach for thermo-mechanical analysis of functionally graded materials. Engineering Analysis with Boundary Elements 32:704-712

Williamson RL, Rabin BH, Drake JT (1993) Finite element analysis of thermal residual stresses at graded ceramic-metal interfaces. part 1: Model description and geometrical effects. Journal of Applied Physics 74:1310-1320

Yang K, Feng WZ, Peng HF, Lv J (2015) A new analytical approach of functionally graded material structures for thermal stress bem analysis. International Communications in Heat and Mass Transfer 62:26-32

Zhang C, Cui M, Wang J, Gao XW, Sladek J, Sladek V (2011) 3d crack analysis in functionally graded materials. Engineering Fracture Mechanics 78:585-604 


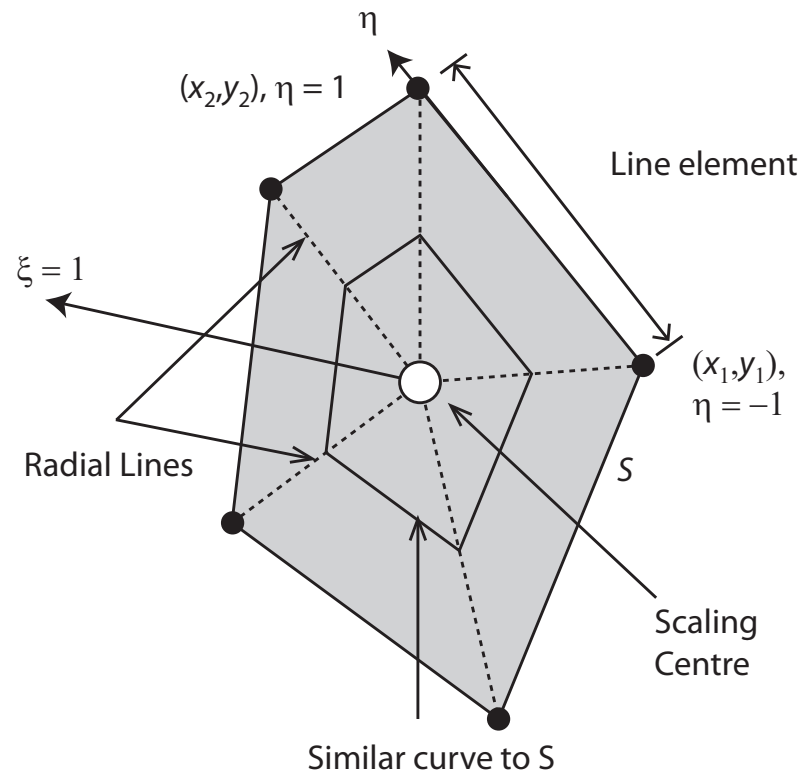

(a)

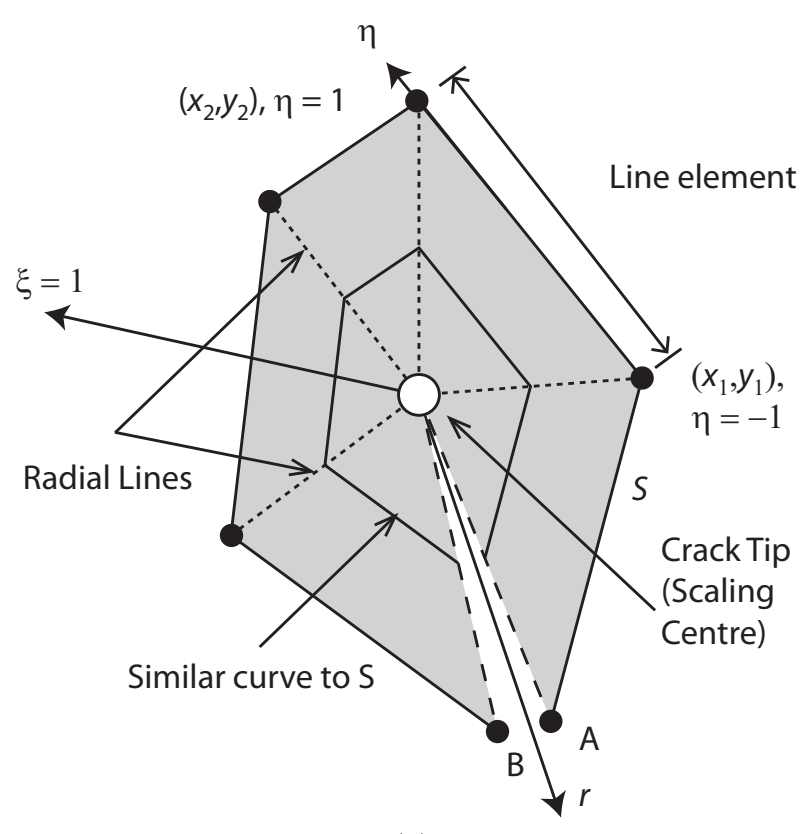

(b)

Fig. 1: A generic polygon modelled by SBFEM: (a) normal polygon; (b) crack polygon. 


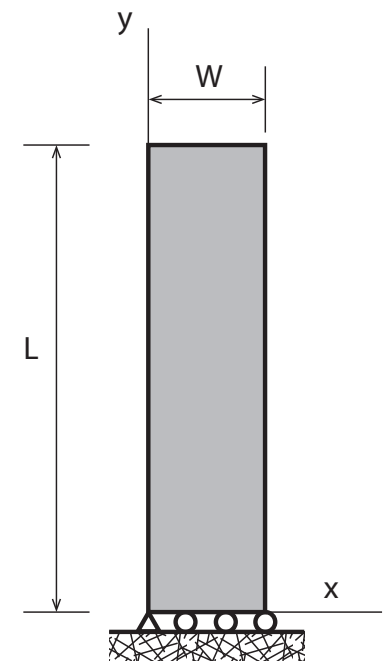

(a)

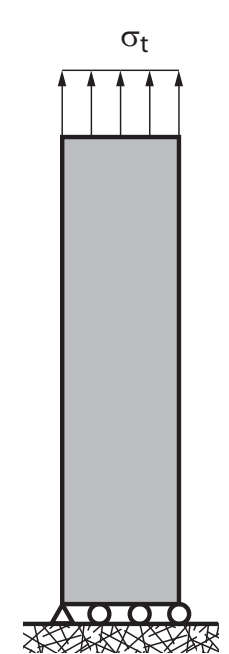

(b)

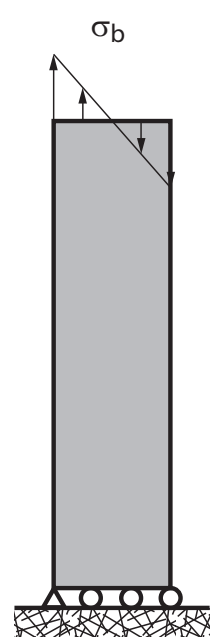

(c)

Fig. 2: A functionally graded plate subjected to various loading conditions: (a) geometry; (b) tensile loading and; (c) bending load. 


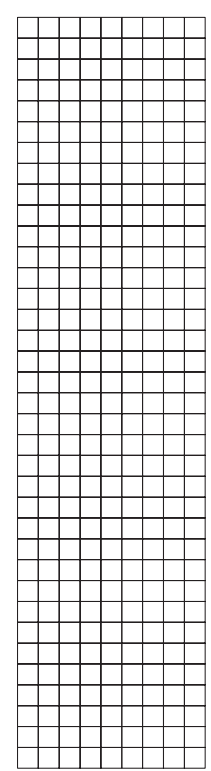

(a)

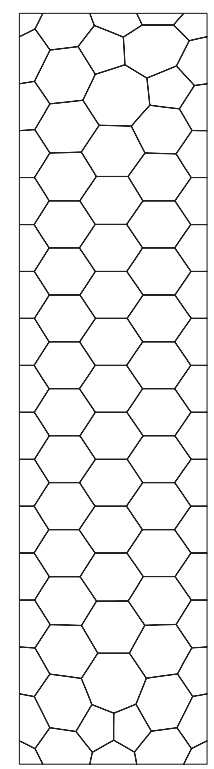

(b)

Fig. 3: Typical meshes used for functionally graded plate problem: (a) regular quadrilateral and; (b) arbitrary sided polygons. 


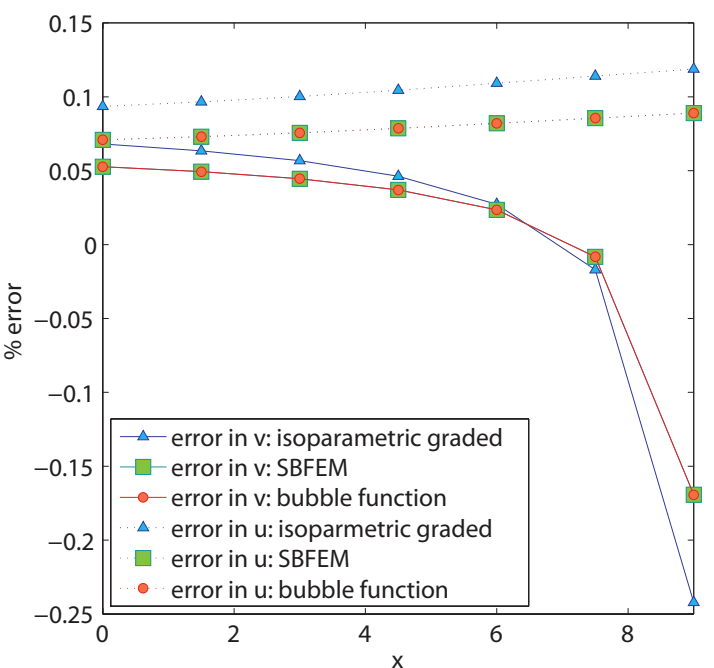

(a)

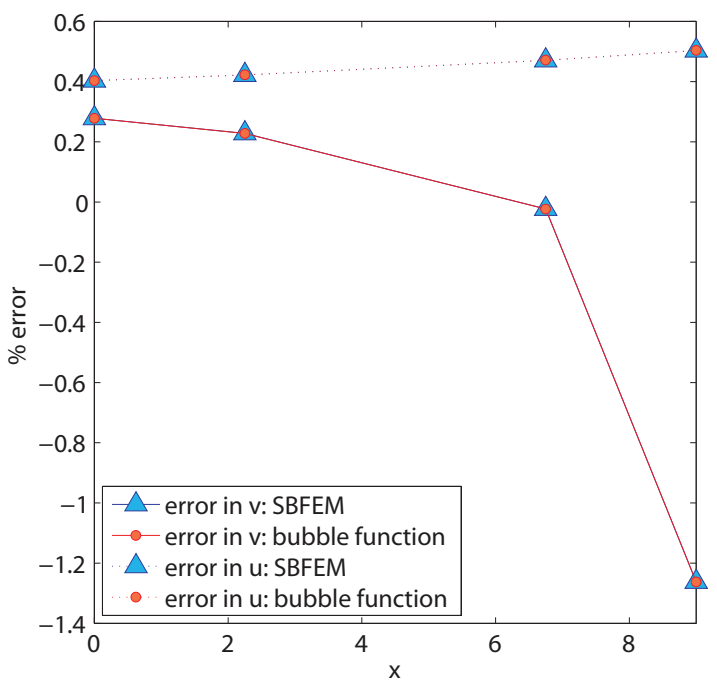

(c)

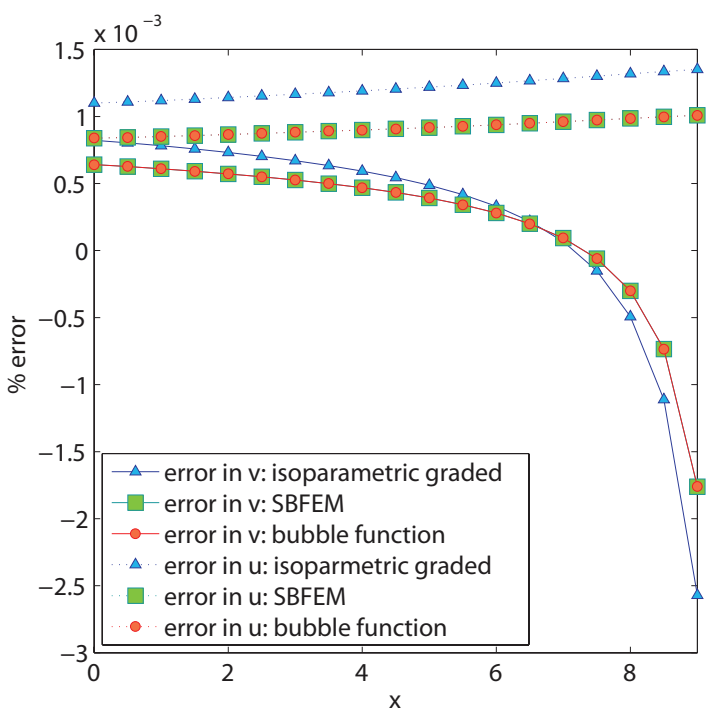

(b)

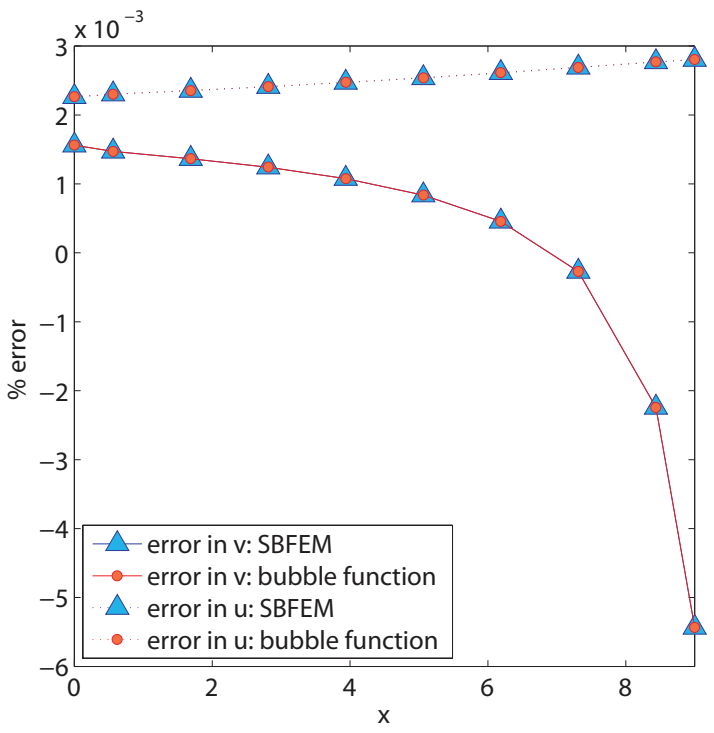

(d)

Fig. 4: Percentage of error in computed nodal displacements at $y=9$ for the isotropic functionally graded plate under tensile loading with exponential material gradient perpendicular to loading direction: (a) $3 \times 12$ regular quadrilateral mesh; (b) $9 \times 36$ regular quadrilateral mesh; (c) $h_{t r i}=4.5$ polygon mesh and; (d) $h_{t r i}=1.125$ polygon mesh. 


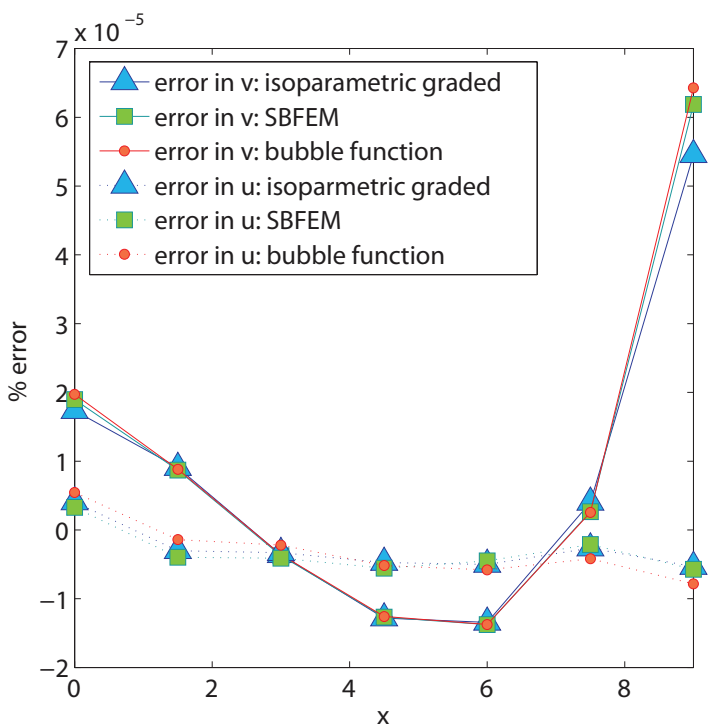

(a)

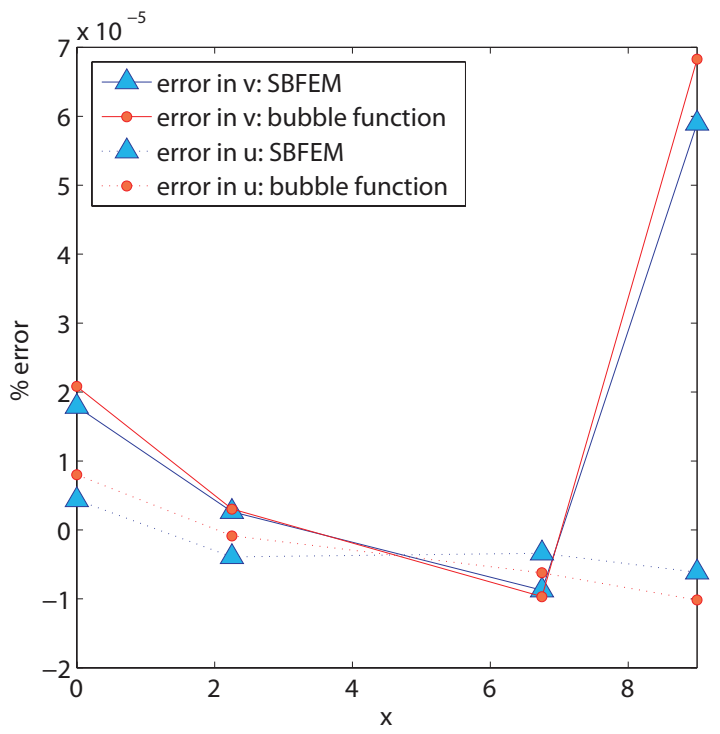

(c)

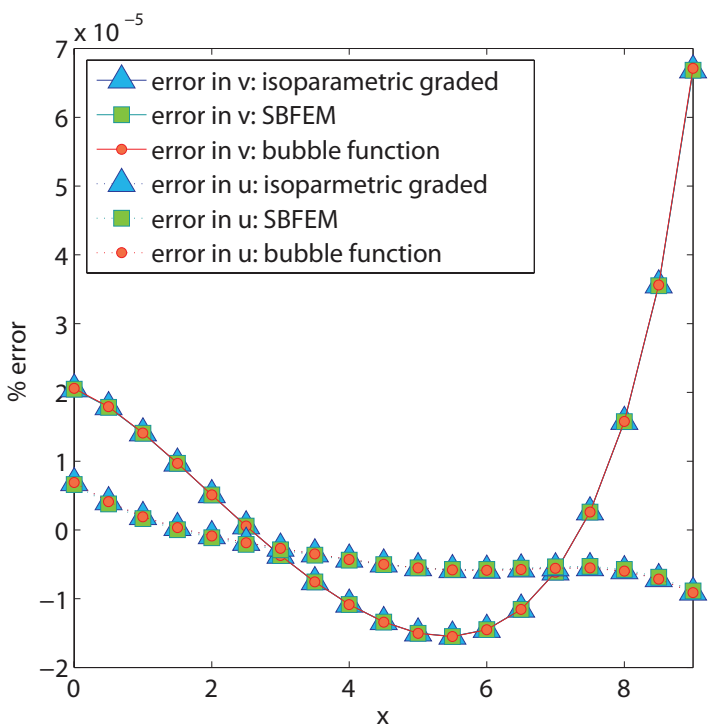

(b)

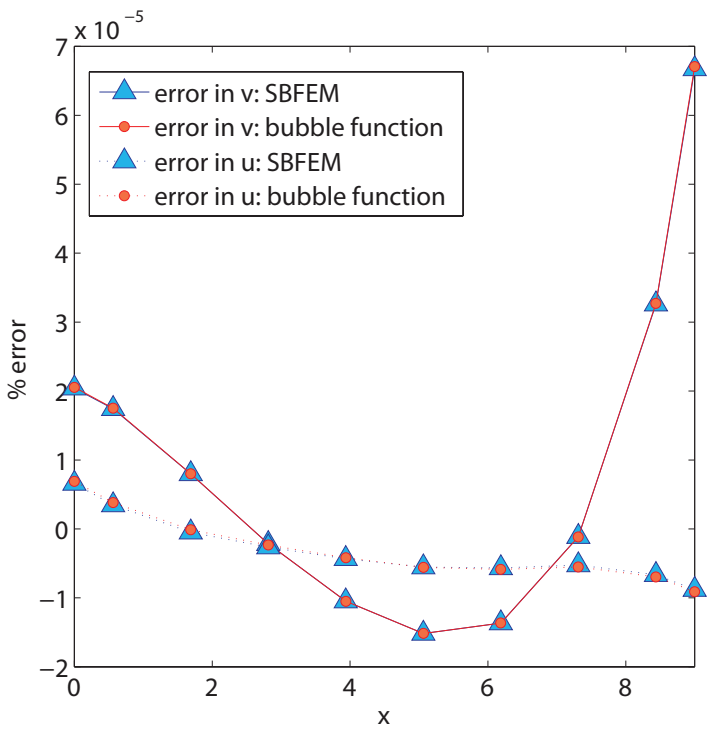

(d)

Fig. 5: Percentage of error in computed nodal displacements at $y=9$ for the isotropic functionally graded plate under tensile loading with linear material gradient perpendicular to loading direction: (a) $3 \times 12$ regular quadrilateral mesh; (b) $9 \times 36$ regular quadrilateral mesh; (c) $h_{t r i}=4.5$ polygon mesh and; (d) $h_{t r i}=1.125$ polygon mesh. 


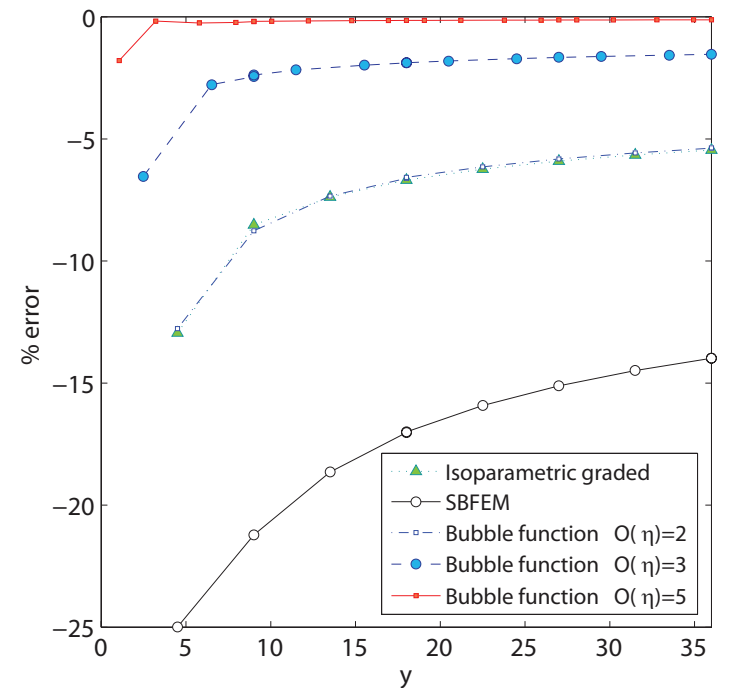

(a)

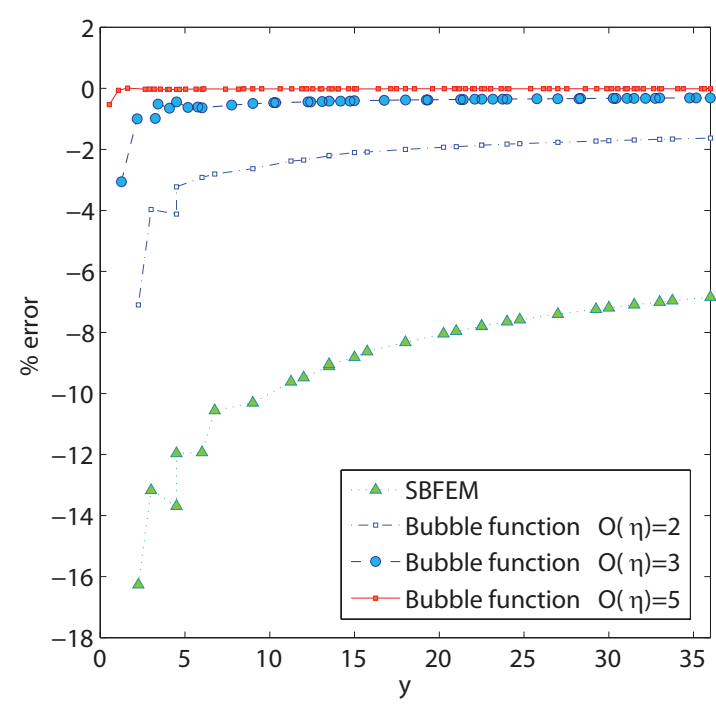

(c)

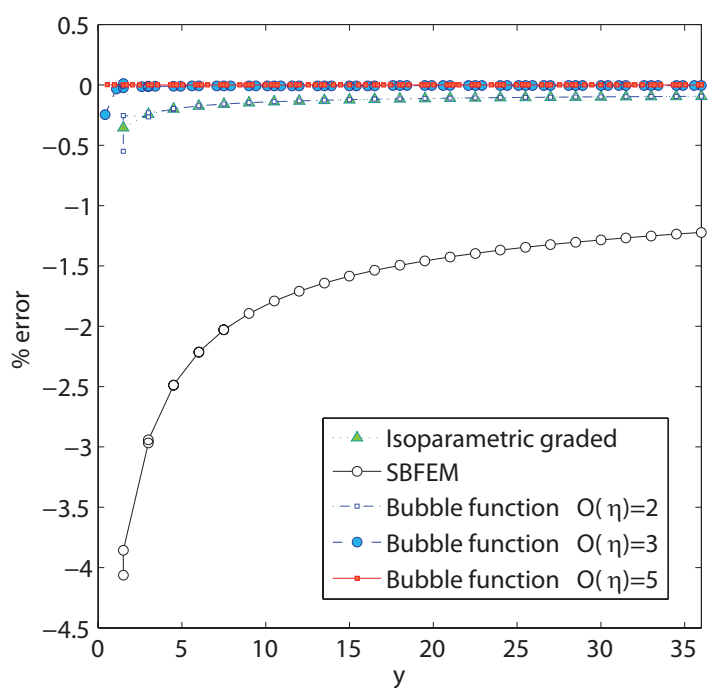

(b)

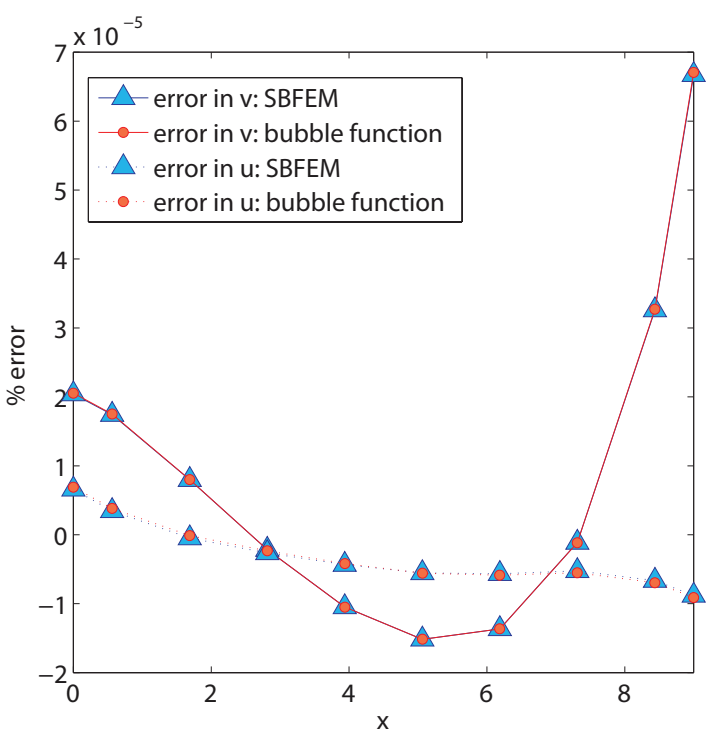

(d)

Fig. 6: Percentage of error in computed nodal displacements at $y=36$ for the isotropic functionally graded plate under tensile loading with material gradient $n=1$ parallel to loading direction: (a) $3 \times 12$ regular quadrilateral mesh; (b) $9 \times 36$ regular quadrilateral mesh; (c) $h_{t r i}=4.5$ polygon mesh and; (d) $h_{t r i}=1.125$ polygon mesh. 


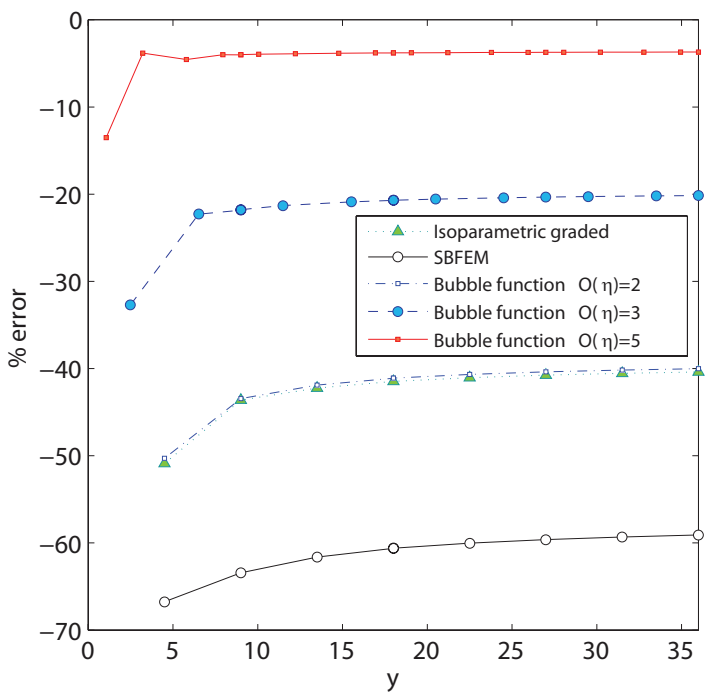

(a)

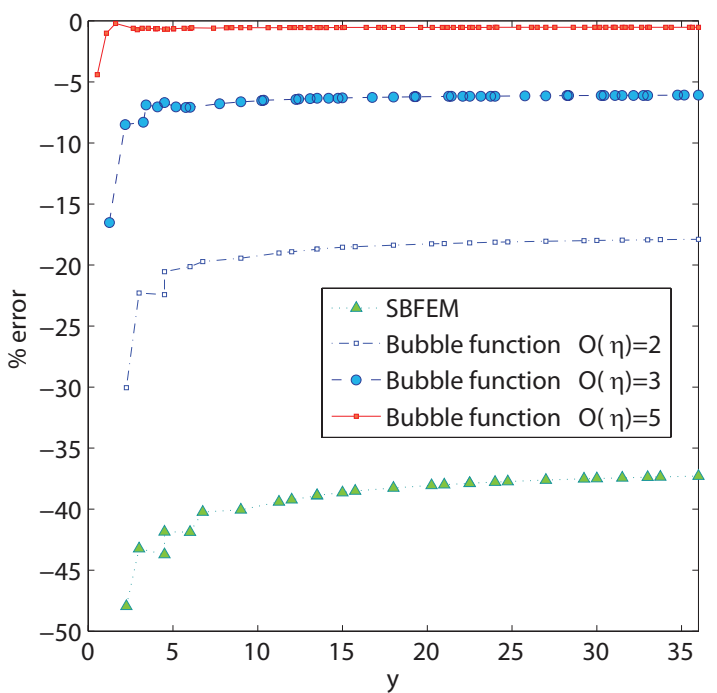

(c)

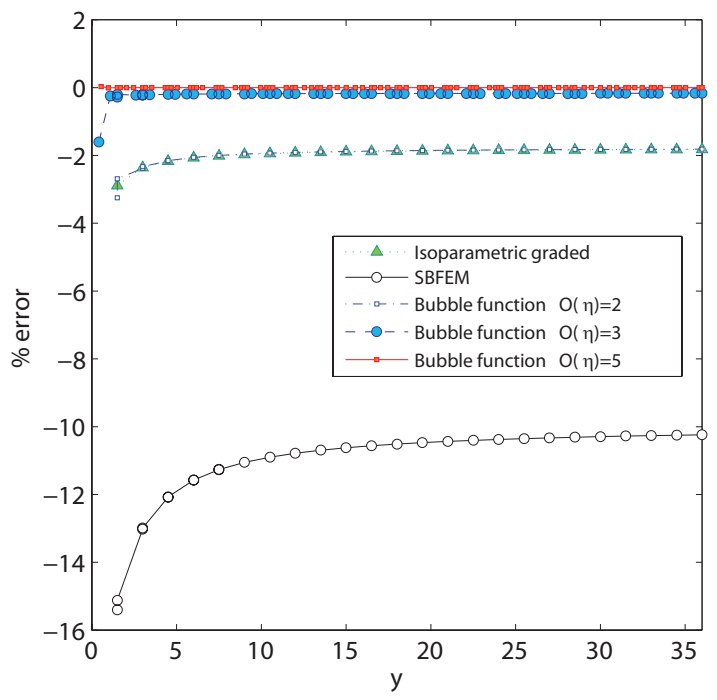

(b)

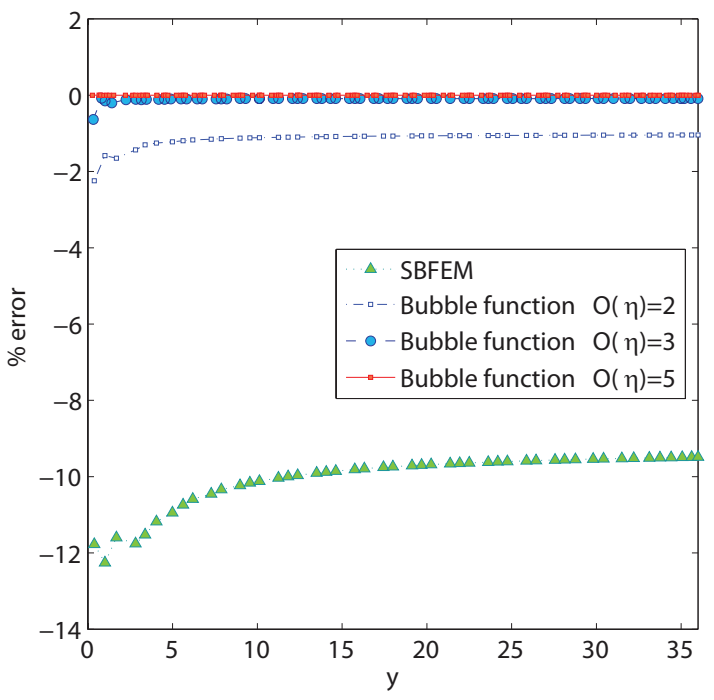

(d)

Fig. 7: Percentage of error in computed nodal displacements at $y=36$ for the isotropic functionally graded plate under tensile loading with with material gradient $n=2$ parallel to loading direction: (a) $3 \times 12$ regular quadrilateral mesh; (b) $9 \times 36$ regular quadrilateral mesh; (c) $h_{t r i}=4.5$ polygon mesh and; (d) $h_{t r i}=1.125$ polygon mesh. 


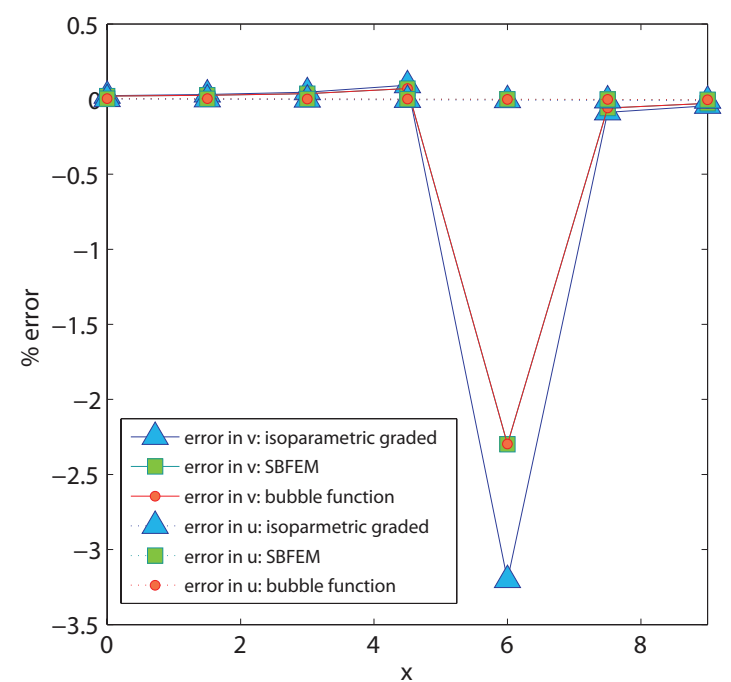

(a)

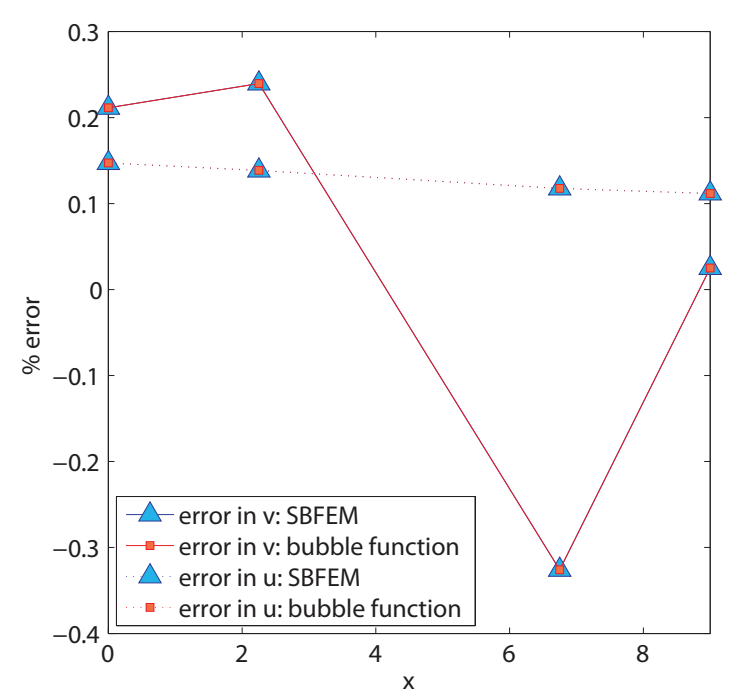

(c)

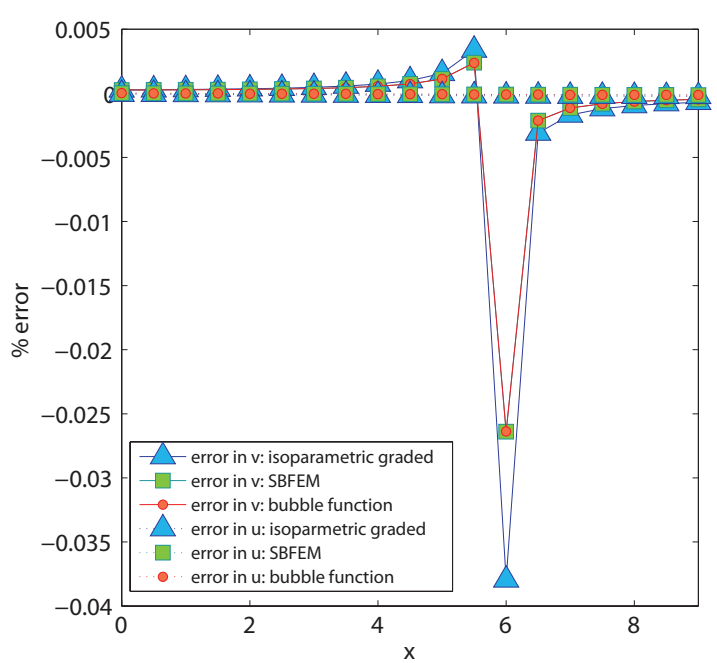

(b)

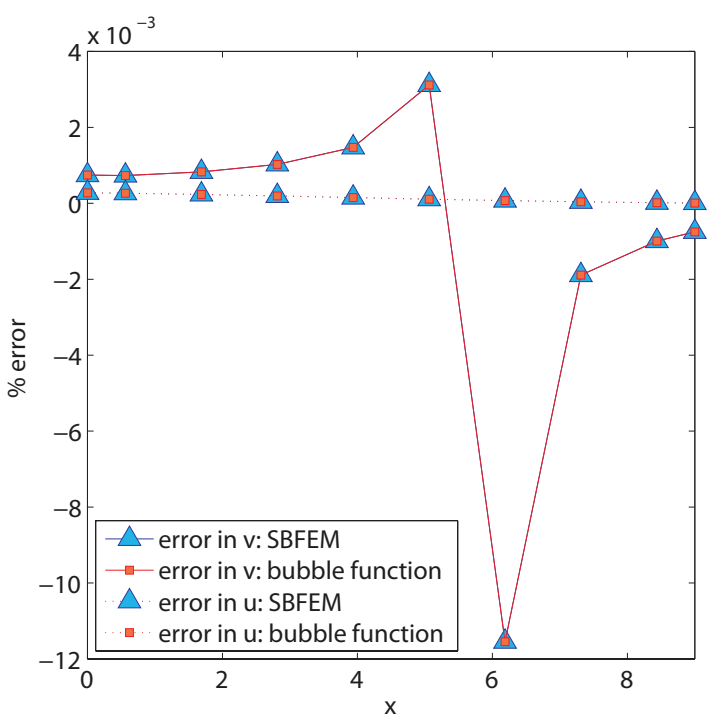

(d)

Fig. 8: Percentage of error in computed nodal displacements at $y=9$ for the isotropic functionally graded plate under bending load with exponential material gradient perpendicular to loading direction: (a) $3 \times 12$ regular quadrilateral mesh; (b) $9 \times 36$ regular quadrilateral mesh; (c) $h_{t r i}=4.5$ polygon mesh and; (d) $h_{t r i}=1.125$ polygon mesh. 


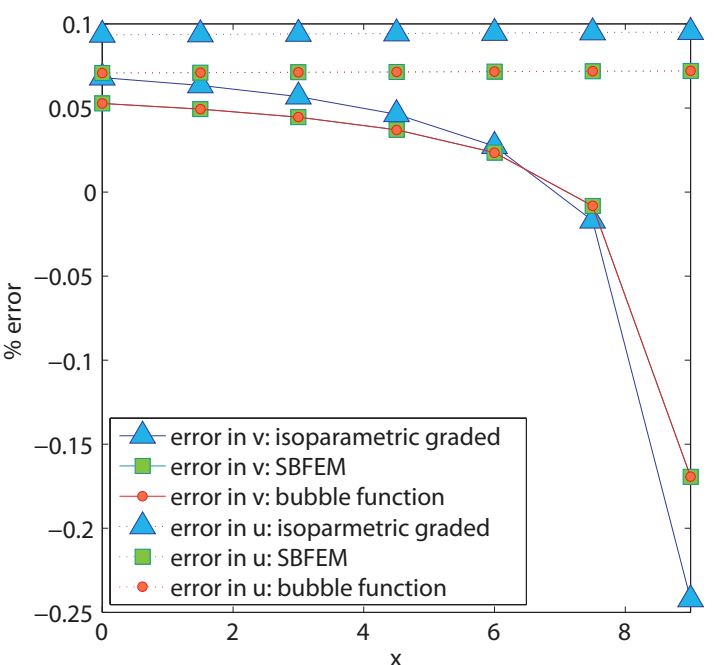

(a)

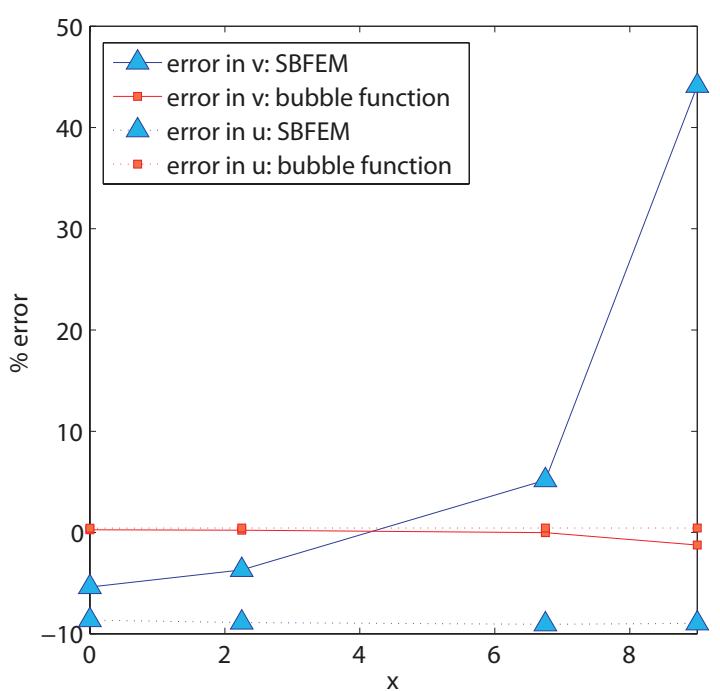

(c)

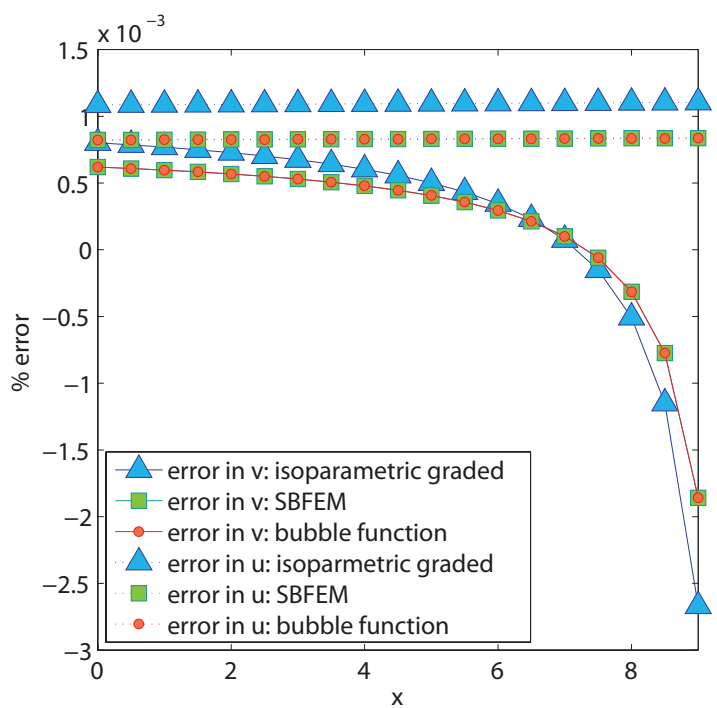

(b)

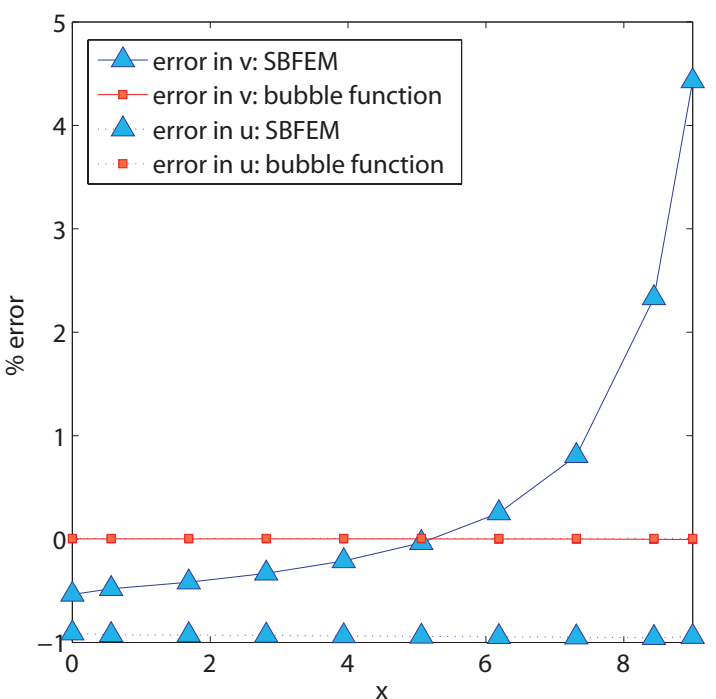

(d)

Fig. 9: Percentage of error in computed nodal displacements at $y=9$ for the orthotropic functionally graded plate under tensile load with exponential material gradient perpendicular to loading direction: (a) $3 \times 12$ regular quadrilateral mesh; (b) $9 \times 36$ regular quadrilateral mesh; (c) $h_{t r i}=4.5$ polygon mesh and; (d) $h_{t r i}=1.125$ polygon mesh. 


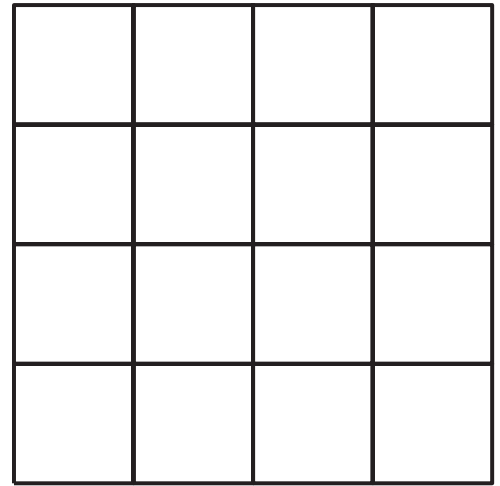

(a)

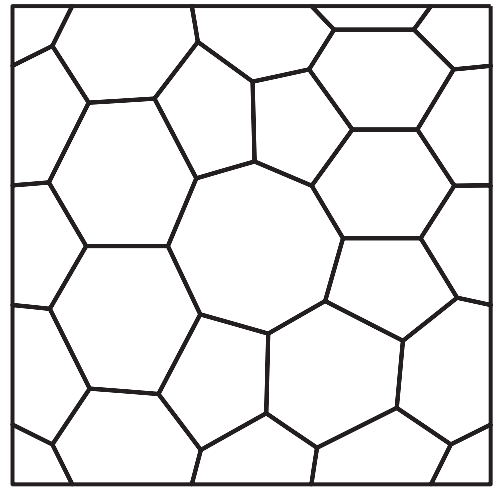

(b)

Fig. 10: Typical meshes used for Poisson problem: (a) regular quadrilateral and; (b) arbitrary sided polygons.

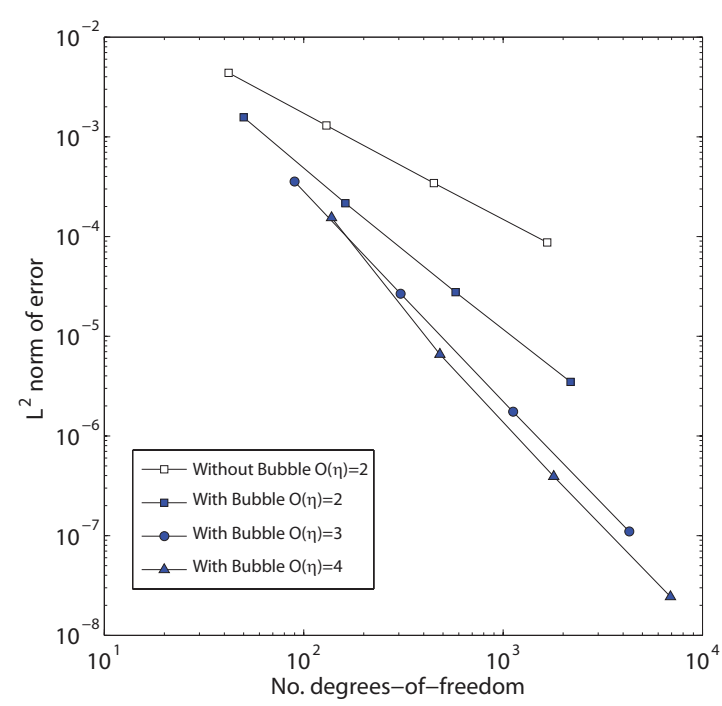

(a)

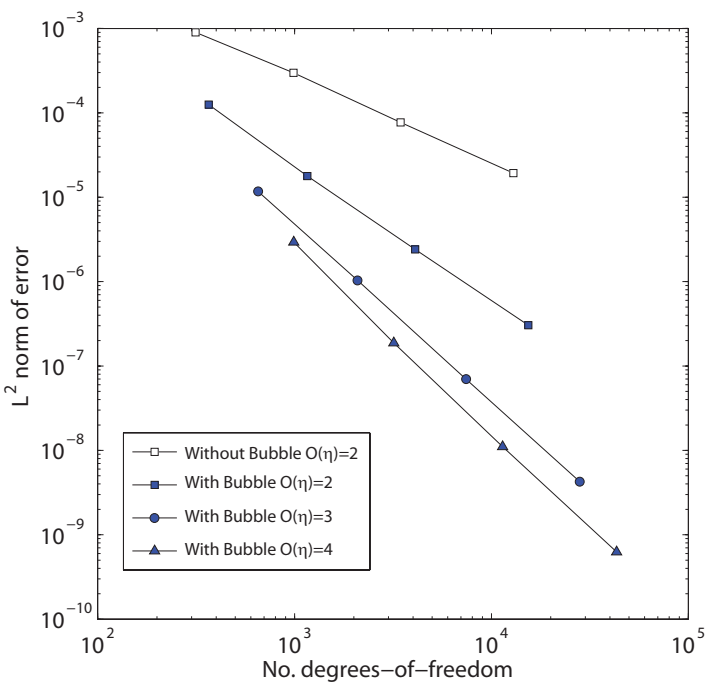

(b)

Fig. 11: Convergence plot for Poisson problem subjected to a polynmial displacement field: (a) regular quadrilateral mesh and (b) polygon mesh. 


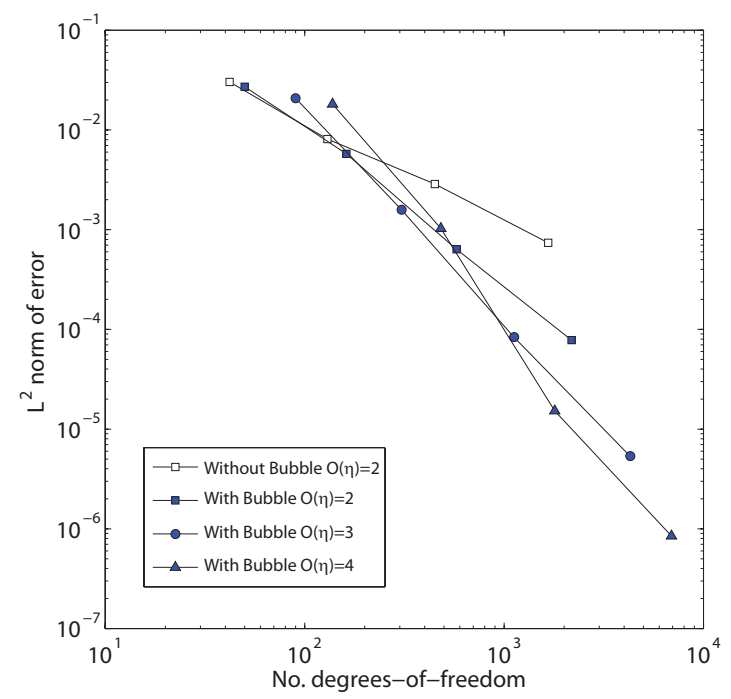

(a)

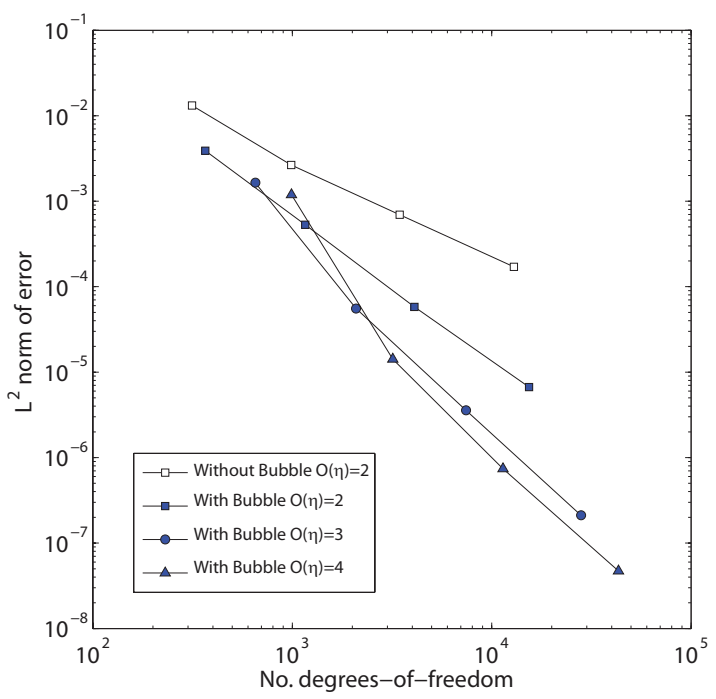

(b)

Fig. 12: Convergence plot for Poisson problem subjected to a trigonometric displacement field: (a) regular quadrilateral mesh and (b) polygon mesh. 


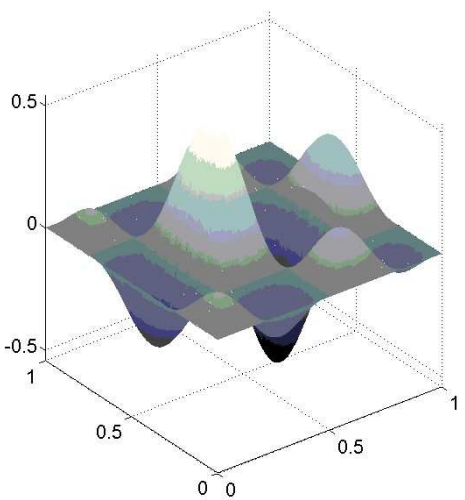

(a)

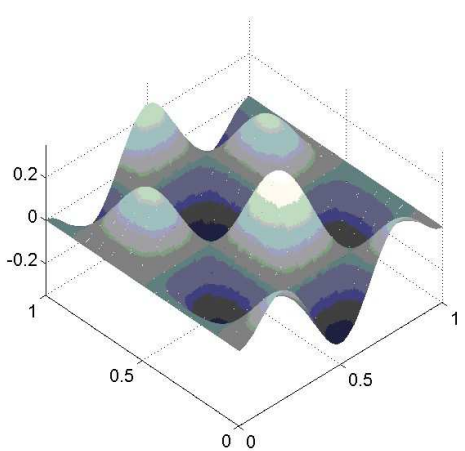

(d)

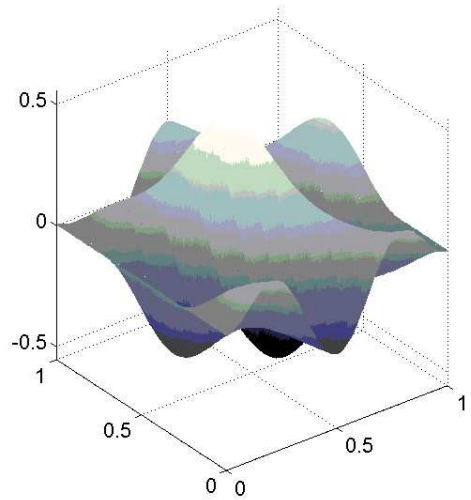

(g)

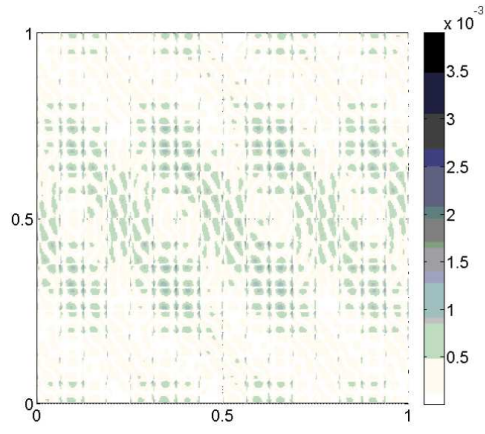

(b)

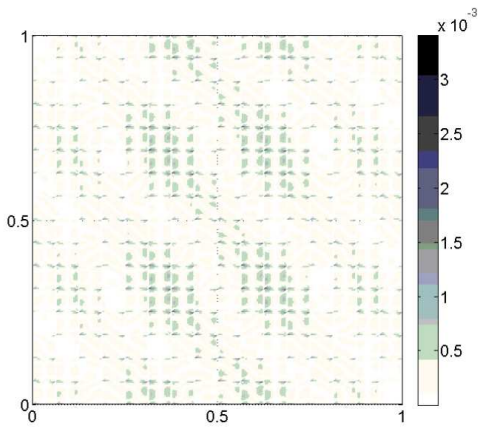

(e)

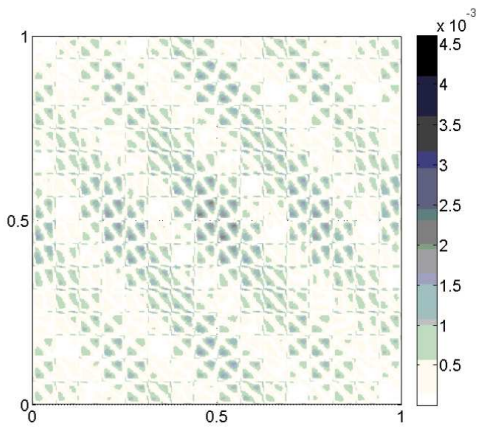

(h)

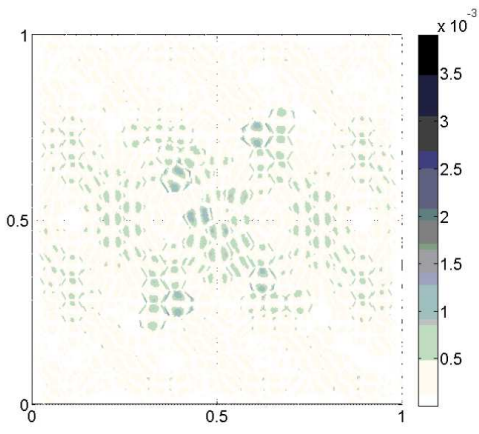

(c)

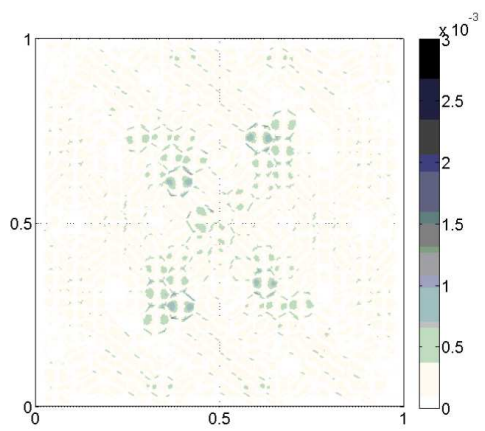

(f)

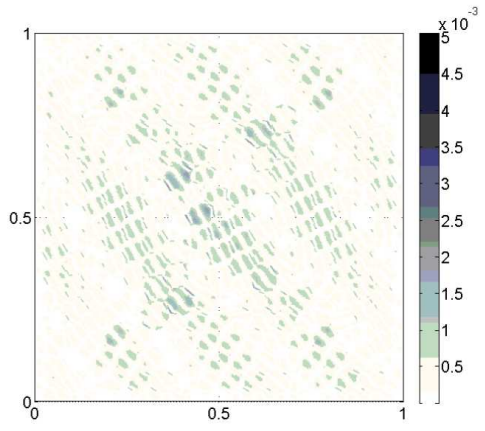

(i)

Fig. 13: Contour plot of strain $\boldsymbol{\epsilon}=\left[\begin{array}{lll}\epsilon_{x x} & \epsilon_{y y} & \gamma_{x y}\end{array}\right]$ and associated errors for Poisson problem with a trigonomeric displacement field obtained from a regular quadrilateral mesh $N=16$ and polygon mesh with $M=16$ using SBFEM with bubble functions of order $O(\eta)=3$ : (a) $\epsilon_{x x}$-plot analytical; (b) error in $\epsilon_{x x}$ - quadrilateral; (c) error in $\epsilon_{y y}$-polygon; (d) $\epsilon_{y y}$-plot analytical; (e) error in $\epsilon_{y y}$ - quadrilateral; (f) error in $\epsilon_{y y}$-polygon; (g) $\gamma_{x y}$-plot analytical; (h) error in $\gamma_{x y}$ - quadrilateral; (i) error in $\gamma_{x y}$-polygon. 


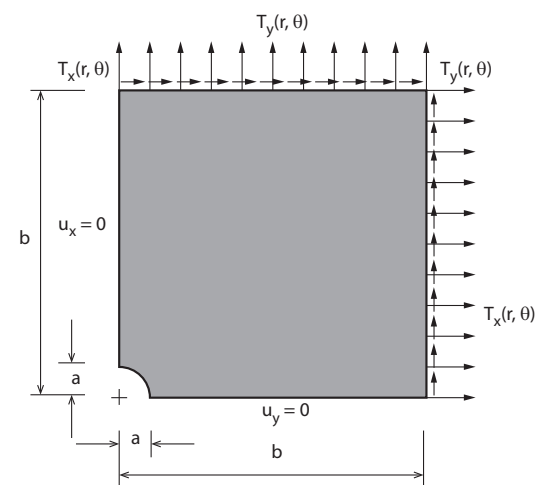

(a)

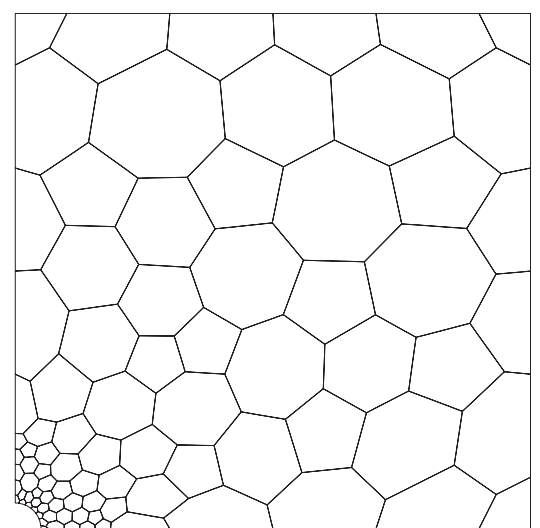

(b)

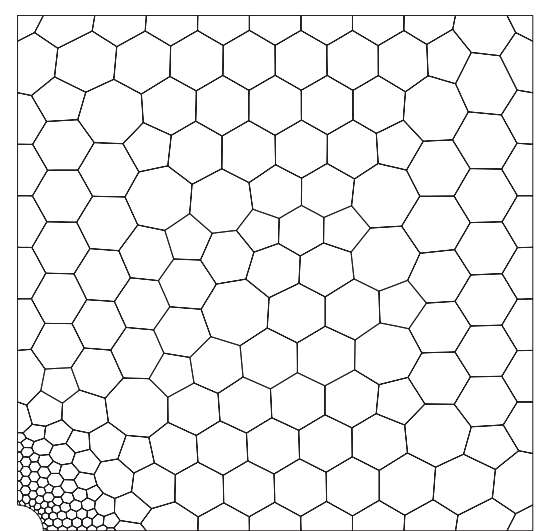

(c)

Fig. 14: Isotropic functionally graded plate with a circular hole subjected to biaxial tension: (a) quarter model of the plate with geometry and boundary conditions; (b) coarse polygon mesh and; (c) fine polygon mesh.

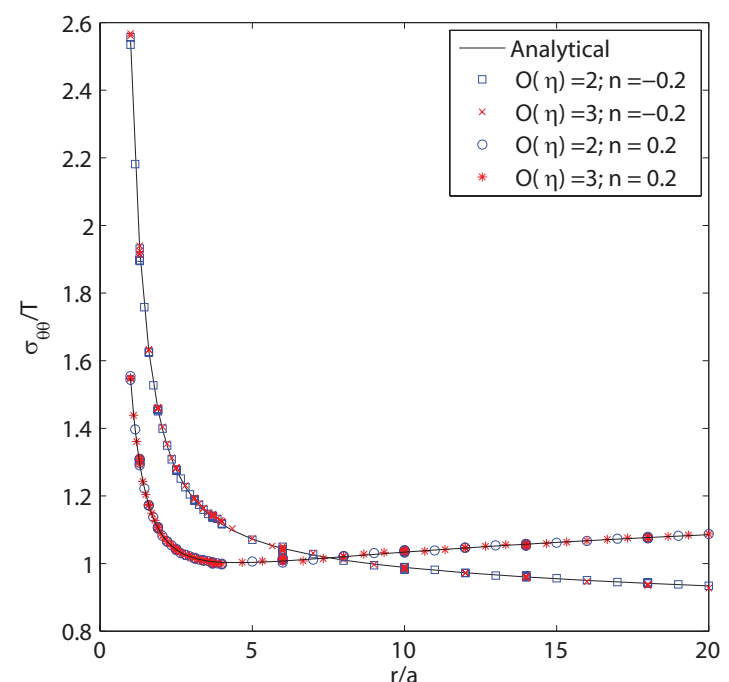

(a)

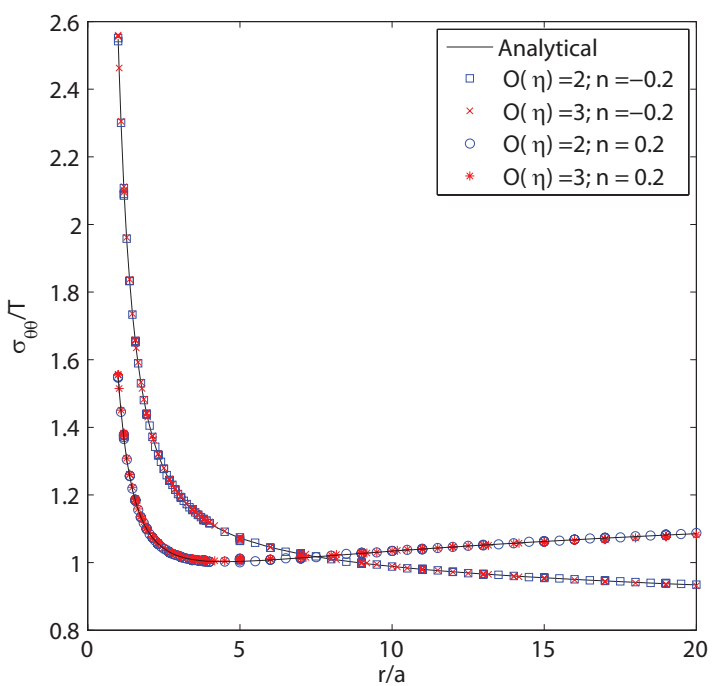

(b)

Fig. 15: Distribution of $\sigma_{\theta \theta} / T$ with respect to distance $r / a$ at $y=0$ for the functionally graded plate with a hole: (a) coarse mesh and; (b) fine mesh. 


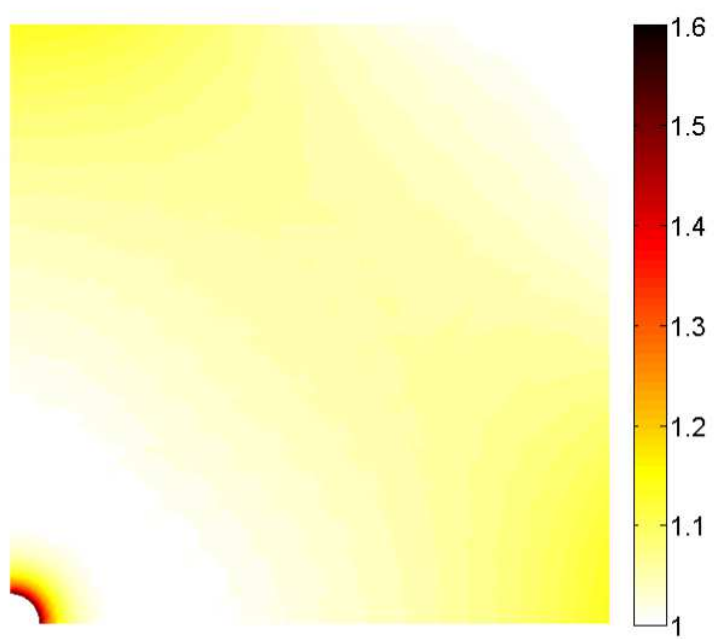

(a)

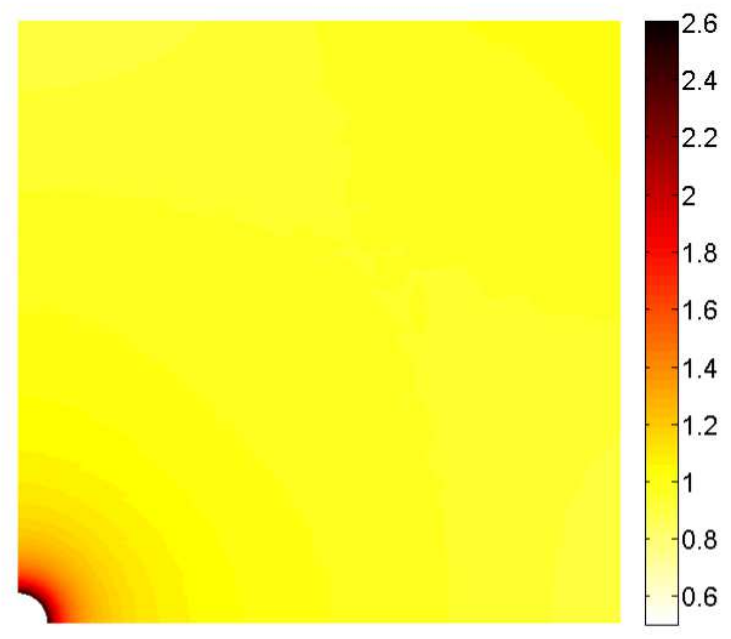

(b)

Fig. 16: Contour plot of $\sigma_{\theta \theta} / T$ for the functionally graded plate with a hole: (a) $n=0.2$; and (b) $n=-0.2$.

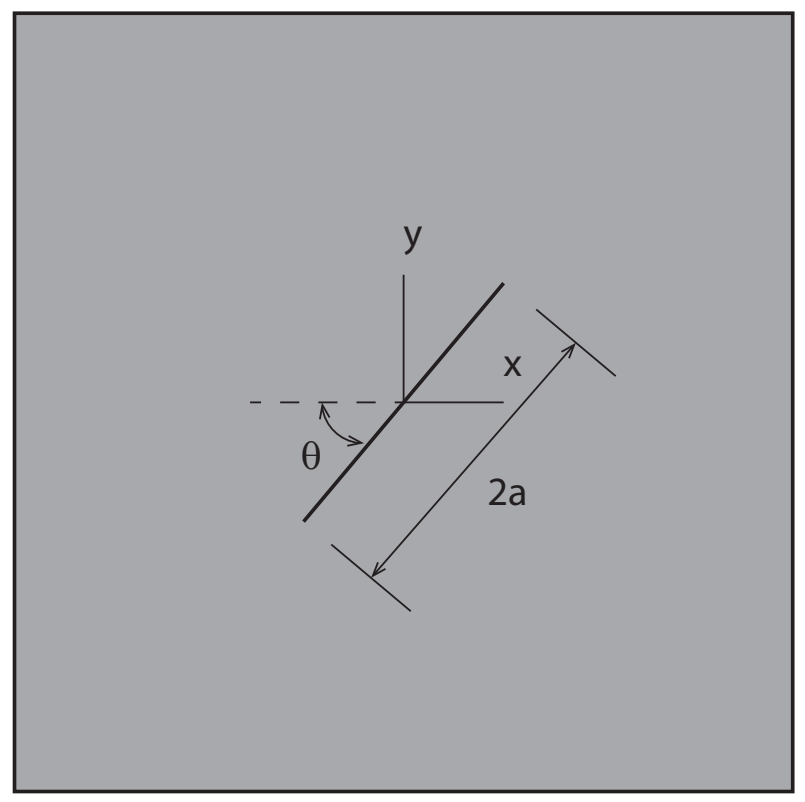

Fig. 17: Functionally graded medium with an internal crack. 


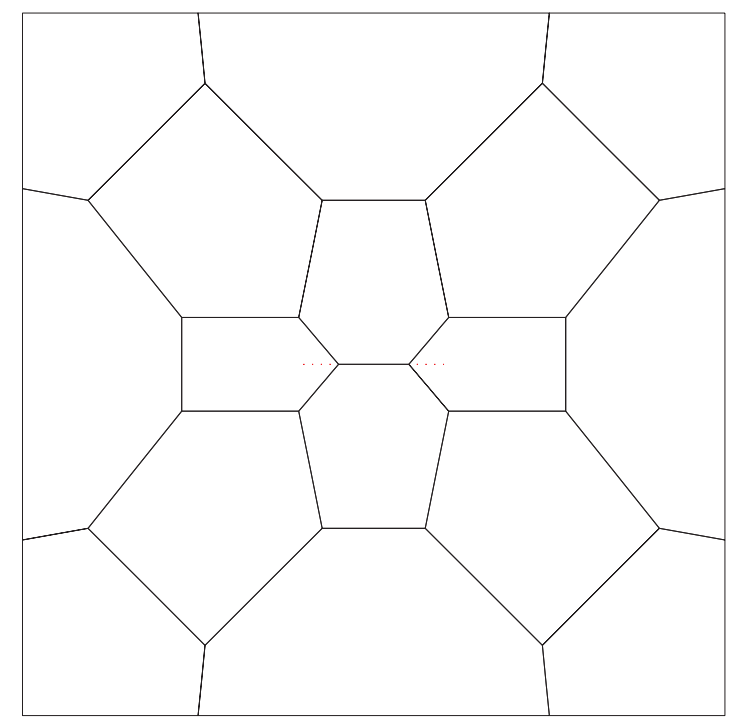

(a) $h=4.0$

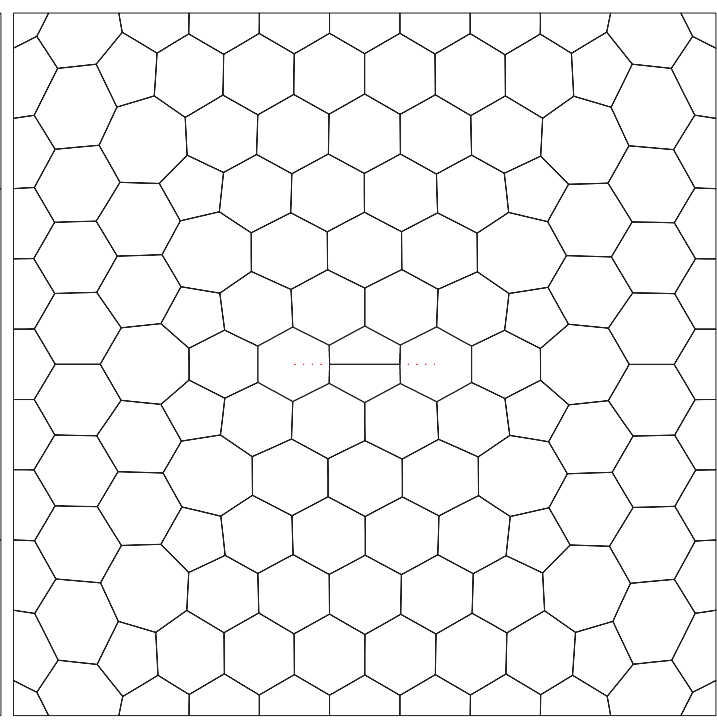

(b) $h=1.0$

Fig. 18: Typical polygon meshes of functionally graded plate with a central crack. 